\title{
Discontinuous approximation of viscous two-phase flow in heterogeneous porous media
}

\author{
Raimund Bürger ${ }^{\mathrm{a}}$, Sarvesh Kumar ${ }^{\mathrm{b}}$, Sudarshan Kumar Kenettinkara ${ }^{\mathrm{c}}$, Ricardo Ruiz-Baier ${ }^{\mathrm{d}}$ \\ ${ }^{a} C I^{2} M A$ and Departamento de Ingeniería Matemática, Universidad de Concepción, \\ Casilla 160-C, Concepción, Chile \\ ${ }^{b}$ Department of Mathematics, Indian Institute of Space Science and Technology, \\ Thiruvananthapuram 695 547, Kerala, India \\ ${ }^{c} C I^{2} M A$, Universidad de Concepción, \\ Casilla 160-C, Concepción, Chile \\ ${ }^{d}$ Mathematical Institute, Oxford University, Andrew Wiles Building, \\ Radcliffe Observatory Quarter, Woodstock Road, OX2 6GG Oxford, UK
}

\begin{abstract}
Runge-Kutta Discontinuous Galerkin (RKDG) and Discontinuous Finite Volume Element (DFVE) methods are applied to a coupled flow-transport problem describing the immiscible displacement of a viscous incompressible fluid in a non-homogeneous porous medium. The model problem consists of nonlinear pressure-velocity equations (assuming Brinkman flow) coupled to a nonlinear hyperbolic equation governing the mass balance (saturation equation). The mass conservation properties inherent to finite volume-based methods motivate a DFVE scheme for the approximation of the Brinkman flow in combination with a RKDG method for the spatio-temporal discretization of the saturation equation. The stability of the uncoupled schemes for the flow and for the saturation equations are analyzed, and several numerical experiments illustrate the robustness of the numerical method.
\end{abstract}

Key words: Two phase flow, Brinkman equations, Runge-Kutta discontinuous Galerkin methods, Stabilization, Finite volume element methods, Discontinuous fluxes 2000 MSC: 76S05, 65M08, 65M60, 65M12

\section{Introduction}

\subsection{Scope}

It is the purpose of this contribution to introduce a new numerical approach for the accurate simulation of viscous two-phase flow (for instance of oil and water) in a heterogeneous porous medium. The flow of the mixture is governed by the Brinkman model while the interaction of the two phases can be described by the fractional flow formalism, which translates into a transport equation of one phase that involves a nonlinear flux function. Since the medium is considered non-homogeneous, not only do the flow properties undergo abrupt changes (as does the permeability of the medium), but also the flux characterization will exhibit discontinuities associated to different nonlinearities adjacent to a discontinuity in the medium.

Specifically, the governing model is defined as follows. Let us consider a mixture of two fluids, a wetting phase and a non-wetting phase (e.g., water and oil) identified by the indices $\mathrm{w}$ and $\mathrm{n}$, with saturations $\phi_{\mathrm{w}}=\phi$ and $\phi_{\mathrm{n}}=1-\phi$, respectively, in a domain $\Omega \subset \mathbb{R}^{2}$. If the fluids are incompressible

Email addresses: rburger@ing-mat.udec.cl (Raimund Bürger), sarvesh@iist.ac.in (Sarvesh Kumar), skumar@ci2ma.udec.cl (Sudarshan Kumar K.), ruizbaier@maths.ox.ac.uk (Ricardo Ruiz-Baier) 
and capillary forces are negligible, then the following model is adequate to describe the viscous motion of the mixture in a porous medium:

$$
\begin{array}{rc}
\partial_{t} \phi+\operatorname{div} \mathbf{F}(\phi, \boldsymbol{u}, \boldsymbol{x})=0 & \text { in } \Omega \times(0, T), \\
\mathbf{K}^{-1}(\boldsymbol{x}) \boldsymbol{u}-\operatorname{div}(\mu(\phi) \boldsymbol{\varepsilon}(\boldsymbol{u})-p \mathbf{I})-\phi \boldsymbol{g}=\mathbf{0} & \text { in } \Omega \times(0, T), \\
\operatorname{div} \boldsymbol{u}=0 & \text { in } \Omega \times(0, T),
\end{array}
$$

supplied with suitable initial and boundary conditions. Here and elsewhere, div and div are used for the standard divergence of an arbitrary vector $\boldsymbol{v} \in \mathbb{R}^{2}$ and matrix $\mathbf{A} \in \mathbb{R}^{2 \times 2}$, i.e.,

$$
\operatorname{div} \boldsymbol{v}:=\sum_{i=1}^{2} \frac{\partial v_{i}}{\partial x_{i}}, \quad \operatorname{div} \mathbf{A}:=\left(\sum_{j=1}^{2} \frac{\partial a_{i j}}{\partial x_{j}}\right)_{1 \leq i \leq 2}
$$

The primal unknowns are the volume average flow velocity of the mixture $\boldsymbol{u}$, the saturation $\phi$, and the pressure field $p$. In addition, $\mu(\phi) \varepsilon(\boldsymbol{u})-p \mathbf{I}$ is the Cauchy stress tensor, $\boldsymbol{\varepsilon}(\boldsymbol{u})=\frac{1}{2}\left(\nabla \boldsymbol{u}+\nabla \boldsymbol{u}^{\mathrm{T}}\right)$ is the infinitesimal rate of strain, $\mu=\mu(\phi)$ is the saturation-dependent viscosity,

$$
\mathbf{F}(\phi, \boldsymbol{u}, \boldsymbol{x})=f(\phi) \boldsymbol{u}+b(\phi) \mathbf{K}(\boldsymbol{x}) \boldsymbol{g}
$$

is a nonlinear flux vector, $\mathbf{K}$ is the permeability tensor of the medium, and $\boldsymbol{g}$ is the gravity acceleration. Moreover, $f$ and $b$ are given fractional flow functions that will be specified later.

We assume that $\mathbf{K}$ is symmetric, uniformly bounded and positive definite, and that $\mu$ and $\mathbf{K}^{-\mathbf{1}}$ satisfy

$$
\begin{array}{r}
\mu, \mu^{\prime} \in \operatorname{Lip}\left(\mathbb{R}_{+}\right) ; \quad \exists \gamma_{1}, \mu_{\min }, \mu_{\max }>0: \forall s \in \mathbb{R}_{+}: \mu_{\min }<\mu(s)<\mu_{\max },\left|\mu^{\prime}(s)\right| \leq \gamma_{1}, \\
\forall \boldsymbol{x} \in \Omega ; \quad \exists k_{1}, k_{2}>0 \text { such that } 0<k_{1} \leq \mathbf{K}^{-1}(x) \leq k_{2},
\end{array}
$$

where the last inequalities are understood in a component-wise sense.

The numerical approximation of the system (1.1) calls for advanced techniques capable to accurately capture flux discontinuities, and robust flow solvers that satisfy discrete maximum principles ( $\phi$ must remain bounded between zero and one), produce divergence-free approximations of velocity, and ensure local mass conservation. It is known that classical methods (e.g. pure upwind finite volumes or primal finite elements and others) do not capture the behavior of the flow near the interface and may yield nonphysical solutions unless some sort of monotonicity-preserving slope limiter or high-order reconstruction is added. Other possible remedies include multi-point flux approximation, high-order DG or other nonconforming methods, phase field models, XFEM, or level set strategies. Regarding the flow equations (in this case, of Brinkman type), we are interested in accurate methods that would permit a natural development of error estimates (therefore associated with finite element formulations), that are mass conservative by construction (therefore related to mixed formulations, or to pure finite volume schemes), and which could easily handle unstructured meshes. These aspects are the prime motivation for proposing Discontinuous Finite Volume Element (DFVE) methods for the approximation of the Brinkman equations 1.1b, 1.1c and Runge-Kutta Discontinuous Galerkin (RKDG) approximation of the saturation equation (1.1a). The main novelty of this paper is the particular numerical treatment of the two-phase flow equations (1.1) through the implementation of the RKDG scheme together with the DFVE method and the corresponding stability analysis. Since we have included the effect of gravity and the medium is heterogeneous, the flux function in $1.1 \mathrm{a}$ is non-monotone in $\phi$ and discontinuous in $\boldsymbol{x}$. These properties require an appropriate choice of the numerical flux in the RKDG formulation. Showing the robustness of numerical results that arise from incorporating the so-called DFLU flux of [2] into the RKDG formulation further adds to the novelty.

\subsection{Related work}

The Brinkman model exhibits the well-known advantage that one can represent both Stokes and Darcy flows without imposing explicit interface (e.g. Beavers-Joseph-Saffman) conditions. The latter 
are not necessarily consistent with mixture theory, and are quite difficult to treat numerically. In fact, several vectorial and scalar Lagrange multipliers need to be incorporated to impose energy conservation, as is done, for instance, in [3, 16, 28].

The model (1.1) is similar to the continuum-based description of sedimentation and consolidation of suspended particles recently discretized by FVE-related methods [14, 41. In turn, multiscale FVE methods were applied in [22, 25] for the simulation of two-phase flow in porous media. An adaptive FVE was proposed in [36] for a steady convection-diffusion-reaction problem. In [12, 30] the authors propose and analyze unified DFVE methods for Stokes-transport and Darcy-transport problems, respectively; in 14 an axisymmetric sedimentation problem is discretized with a combination of continuous FVE and DFVE for Stokes and a degenerate parabolic equation, continuous FVE-based formulations for coupled Darcy and transport were proven to satisfy discrete maximum principles [23] and applied to NavierStokes-transport couplings in [37, 41; ; and a hybrid mixed FE-DFVE has been recently introduced in [40] for a larger class of multiphase flows in rigid and compliant porous media.

On the other hand, RKDG methods were developed to generate physically relevant numerical solutions to nonlinear hyperbolic conservation laws (see e.g. [18, 19, 20] and the references therein). The most attractive features of RKDG methods include high-order accuracy, nonlinear stability, shock capturing, and the capability of handling complicated geometries and boundary conditions. In addition, generalized "slope limiting" techniques for multidimensional conservation laws [18] have also achieved element-wise mass conservation, prevented spurious oscillations, and yielded nonlinear stability of the underlying scheme. More recently, in [45] a related method was proposed that requires the computed RKDG solution in a cell to satisfy additional conservation constraints in adjacent cells. It was shown that the new formulation leads to a CFL condition that is less restrictive than that of the original RKDG method. For a survey on RKDG methods and related schemes for time-dependent problems we refer to e.g. 42 .

Focusing on the approximation of discontinuous fluxes, one can find many other competing strategies that deal either with the pure transport equation or with the coupling with the description of the flow. These include high order schemes [11, XFEM formulations to capture discontinuities [24], high-order DG discretizations [7, level set methods, phase field schemes [44, multi-point flux approximation methods [17, artificial viscosity [6], and multiresolution-based adaptivity [13].

\subsection{Outline of the paper}

The remainder of the paper is organized as follows. In Section 2 we collect some preliminaries, including a description of the fractional flow model (Sect. 2.2), a statement of the initial and boundary conditions for (1.1) (Sect.2.3), and the weak formulation of the initial-boundary value problem (Sect. 2.4). The spatio-temporal discretization, namely the DFVE-RKDG method, is introduced in Section 3 , starting with the FVE formulation for the flow equations $(1.1 \mathrm{~b})$ and $(1.1 \mathrm{c})$ (Sect. 3.1). Based on this formulation, we derive in Section 3.2 error estimates for the velocity $\boldsymbol{u}$ and the pressure $p$ in terms of the saturation $\phi$. We then introduce the DG spatial discretization of the saturation equation (Sect. 3.3). The latter needs to handle discontinuities in $\mathbf{K}(\boldsymbol{x})$, and therefore in $\mathbf{F}(\phi, \boldsymbol{u}, \boldsymbol{x})$, with respect to $\boldsymbol{x}$, where we assume that the discontinuities are aligned with the edges of the mesh. By suitable quadrature formulas we generate, in Section 3.4 the fully discrete version of the RKDG formulation for 1.1a. In Section 3.5 we define a numerical flux that emerges from the modified Godunov flux reconstruction advanced in [1] for scalar one-dimensional conservation laws with discontinuous flux. Next, in Section 3.6, we recall the limiter for triangular finite elements from 19] that is introduced to prevent oscillations that possibly arise with high-order DG formulations. Section 4 is devoted to the stability analysis of the resulting RKDG scheme for 1.1a), with the result that under a suitable CFL condition that scheme produces numerical approximations that assume values in $[0,1]$ only, in agreement with $\phi_{\mathrm{n}} \geq 0, \phi_{\mathrm{w}} \geq 0$ and $\phi_{\mathrm{n}}+\phi_{\mathrm{w}}=1$. In Section 5 numerical examples are presented. Example 1 (Sect. 5.1) is a simple test case with a known exact solution that is used herein to assess the accuracy of the DFVE formulation for continuous fluxes. Example 2 (Sect. 5.2) demonstrates the performance of the coupled DFVE-RKDG scheme for the full 
problem 1.1 on a porous square, including a straight flux discontinuity. More involved discontinuities arise in Examples 3 (flow in a quarter-five spot with discontinuous relative and absolute permeability), in Example 4, in which areas of reduced permeability are distributed in a random-wise manner over the whole computational domain, and in Example 5 that illustrates the effect of a high-contrast heterogeneous permability field. Some conclusions are presented in Section 6 .

\section{Preliminaries}

\subsection{Basic notation}

We use standard notation for Sobolev spaces $H^{s}(\Omega)$ defined on any bounded set $\Omega \subset \mathbb{R}^{2}$ and their associated norms $\|\cdot\|_{s, \Omega}$ and seminorms $|\cdot|_{s, \Omega}$ with $s \geq 0$. Similarly, $L^{2}(\Omega)$ denotes the set of all square integrable function defined on $\Omega$ and for this space the norm and inner product are denoted by $\|\cdot\|_{0, \Omega}$ and $(., .)_{(\Omega)}$, respectively. We also define the vector counterpart of the Sobolev spaces as $\mathbf{H}^{1}(\Omega):=H_{1}(\Omega)^{2}$ with their associated norms $\|\boldsymbol{v}\|_{s, \Omega}^{2}:=\sum_{i=1}^{2}\left\|v_{i}\right\|_{s, \Omega}^{2}$ and seminorms $|\boldsymbol{v}|_{s, \Omega}^{2}:=\sum_{i=1}^{2}\left|v_{i}\right|_{s, \Omega}^{2}$, respectively, for any $\boldsymbol{v} \in \mathbf{H}^{1}(\Omega)$. In the same way we define $\mathbf{L}^{2}(\Omega):=L^{2}(\Omega)^{2}$ with the norm $\|\boldsymbol{v}\|_{0, \Omega}^{2}:=\sum_{i=1}^{2}\left\|v_{i}\right\|_{0, \Omega}^{2}$ for $\boldsymbol{v} \in \mathbf{L}^{2}(\Omega)$. Further, we stress that the same definitions remain true if we replace $\Omega$ by any of its subsets.

\subsection{Fractional flow model}

We assume that the fractional flow functions $f$ and $b$ are given by

$$
f(\phi)=\frac{\lambda_{\mathrm{w}}(\phi)}{\lambda_{\mathrm{w}}(\phi)+\lambda_{\mathrm{n}}(\phi)}, \quad b(\phi)=f(\phi) \lambda_{\mathrm{n}}(\phi) \Delta \rho,
$$

where $\lambda_{\mathrm{w}}, \lambda_{\mathrm{n}}$ are the mobilities of the phases given by

$$
\lambda_{\mathrm{w}}(\phi):=\frac{K_{\mathrm{w}}(\phi)}{\mu_{\mathrm{w}}}, \quad \lambda_{\mathrm{n}}(\phi):=\frac{K_{\mathrm{n}}(\phi)}{\mu_{\mathrm{n}}},
$$

$\Delta \rho:=\rho_{\mathrm{n}}-\rho_{\mathrm{w}}$, where $\rho_{\mathrm{n}}$ and $\rho_{\mathrm{w}}$ are the densities, and $K_{\mathrm{w}}$ and $K_{\mathrm{n}}$ are the relative permeabilities of phase $\mathrm{w}$ and $\mathrm{n}$, respectively.

If the medium is non-homogeneous, for instance due to fractures characterized by sharp localized discontinuities or due to abrupt changes from one rock type to another, then $\mathbf{K}, K_{\mathrm{w}}$ and $K_{\mathrm{n}}$ will typically depend discontinuously on $\boldsymbol{x}$. This assumption implies that the fractional flow functions $f$ and $b$, and consequently the flux $\mathbf{F}$, will also be discontinuous. In addition, the coupling conditions at the interface between two kinds of media typically consist in prescribing continuity of the normal flux across the interface. However, since the algebraic definitions of the flux as a function of saturation differ to both sides of the interface, the saturation will in general be discontinuous across that interface [24].

\subsection{Initial and boundary conditions}

The domain boundary is typically disjointly partitioned into a inlet section, outlet and walls $\partial \Omega=$ $\Gamma_{\text {in }} \cup \Gamma_{\text {out }} \cup \Gamma_{\text {wall }}$. The system $[1.1$ is then supplemented with the following set of boundary conditions

$$
\begin{array}{lll}
\boldsymbol{u}=\boldsymbol{u}_{\mathrm{in}}, & \phi=\phi_{\mathrm{in}}, & \text { on } \Gamma_{\text {in }} \times(0, T), \\
p=p_{\text {out }}, & \mathbf{F} \cdot \boldsymbol{n}=0, & \text { on } \Gamma_{\text {out }} \times(0, T), \\
\boldsymbol{u} \cdot \boldsymbol{n}=0, & \mathbf{F} \cdot \boldsymbol{n}=0, & \text { on } \Gamma_{\text {wall }} \times(0, T)
\end{array}
$$

along with the initial data

$$
\boldsymbol{u}(0)=\boldsymbol{u}_{0}, \quad \phi(0)=\phi_{0} \quad \text { on } \Omega \times\{0\} .
$$

For sake of clarity, instead of using (2.1) we will restrict the presentation to homogeneous Dirichlet data for velocity and saturation on the whole boundary:

$$
\boldsymbol{u}=\mathbf{0}, \quad \phi=0, \quad \text { on } \Gamma \times(0, T) .
$$



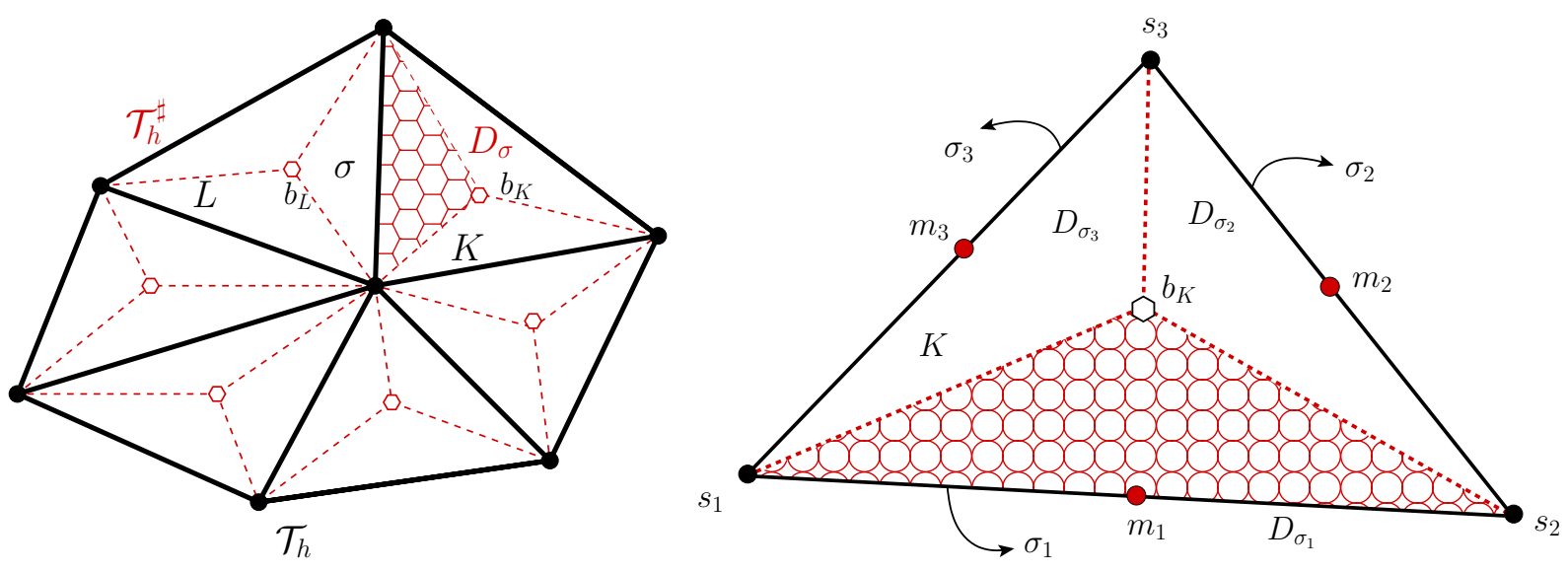

Figure 1: Left: Triangular elements in $\mathcal{T}_{h}$ (solid lines) and diamonds in $\mathcal{T}_{h}^{\sharp}$ (dashed lines). Right: Triangular element $K \in \mathcal{T}_{h}$ (solid lines) and three diamonds $D_{\sigma_{j}}$ (dashed lines). One diamond is filled with a pattern for illustration.

\subsection{Weak formulation}

Multiplying (1.1a by $\varphi \in H_{0}^{1}(\Omega)$ and $1.1 \mathrm{~b}$ by $\boldsymbol{v} \in \mathbf{H}_{0}^{1}(\Omega)$ and using the Gauss divergence theorem yields the following weak formulation for (1.1):

For $0<t<T$, find $(\boldsymbol{u}(t), p(t), \phi(t)) \in \mathbf{H}_{0}^{1}(\Omega) \times L_{0}^{2}(\Omega) \times H_{0}^{1}(\Omega)$ such that

$$
\begin{array}{rlrl}
\left(\partial_{t} \phi, \varphi\right)_{\Omega}-(\mathbf{F}(\boldsymbol{u}, \phi), \nabla \varphi)_{\Omega} & =0 & & \forall \varphi \in H_{0}^{1}(\Omega), \\
a(\boldsymbol{u}, \boldsymbol{v})+c(\boldsymbol{u}, \boldsymbol{v} ; \phi)+b(\boldsymbol{v}, p)-d(\phi, \boldsymbol{v}) & =0 & & \forall \boldsymbol{v} \in \mathbf{H}_{0}^{1}(\Omega), \\
b(\boldsymbol{u}, q) & =0 & \forall q \in L_{0}^{2}(\Omega), \\
\phi(0) & =\phi_{0} & & \text { a.e. in } \Omega,
\end{array}
$$

where $\mathbf{H}_{0}^{1}(\Omega):=\left\{\boldsymbol{v} \in \mathbf{H}^{1}(\Omega):\left.\boldsymbol{v}\right|_{\Gamma}=0\right\}, L_{0}^{2}(\Omega):=\left\{q \in L^{2}(\Omega): \int_{\Omega} q \mathrm{~d} \boldsymbol{x}=0\right\}, H_{0}^{1}(\Omega):=\left\{s \in H^{1}(\Omega)\right.$ : $\left.\left.s\right|_{\Gamma}=0\right\}$ and the involved forms are defined as

$$
\begin{aligned}
a(\boldsymbol{u}, \boldsymbol{v}) & :=\left(\mathbf{K}^{-1}(\boldsymbol{x}) \boldsymbol{u}, \boldsymbol{v}\right)_{\Omega}, \quad c(\boldsymbol{u}, \boldsymbol{v} ; \phi)=(\mu(\phi) \boldsymbol{\varepsilon}(\boldsymbol{u}), \boldsymbol{\varepsilon}(\boldsymbol{v}))_{\Omega}, \\
b(\boldsymbol{v}, q) & :=-(q, \operatorname{div} \boldsymbol{v})_{\Omega}, \quad d(\phi, \boldsymbol{v}):=(\phi \boldsymbol{g}, \boldsymbol{v})_{\Omega} .
\end{aligned}
$$

The existence of weak solutions to this problem has been established in [21].

\section{The discrete formulation}

\subsection{Finite volume elements for the flow equations}

Let $\mathcal{T}_{h}$ be a regular, quasi-uniform partition of the domain $\Omega$ formed by closed triangles. For each triangular element $K$, we denote by $\partial K$ and $h_{K}$ its boundary and diameter, respectively. Each face $\sigma$ between two neighboring elements $K$ and $L$ has diameter $h_{\sigma}$ (see Figure 1, left). The set of all faces in $\mathcal{T}_{h}$ is denoted by $\mathcal{E}_{h}, \mathcal{E}_{h}^{\Gamma}$ is its restriction to boundary faces, and $s_{j}, j=1, \ldots, N_{h}$, are the vertices of triangular mesh. Corresponding to the primal mesh $\mathcal{T}_{h}$ we also define the dual mesh $\mathcal{T}_{h}^{\sharp}$ consisting of all sub-triangles (denoted $D_{\sigma}$ ) obtained as follows: we divide each triangle $K \in \mathcal{T}_{h}$ into three sub-triangles by connecting the barycenter of each original triangle to the vertices of that triangle, see Figure 1.

Now, let $\boldsymbol{n}_{K, \sigma}$ denote the outward vector of $K \in \mathcal{T}_{h}$ normal to $\sigma \subset \partial K$. For a scalar function $q \in L^{2}(\Omega)$ we let $\llbracket q \rrbracket_{\sigma}:=\left.q\right|_{K}-\left.q\right|_{L}$ denote a scalar jump across $\sigma=\bar{K} \cap \bar{L}$, and

$$
\llbracket q \boldsymbol{n}_{K, \sigma} \rrbracket_{\sigma}:=\left.q\right|_{\partial K} \boldsymbol{n}_{K, \sigma}-\left.q\right|_{\partial L} \boldsymbol{n}_{L, \sigma}
$$


denotes a vector jump across $\sigma$. By $\{q\}_{\sigma}$ we denote its average value on $\sigma$, and if $\sigma \in \mathcal{E}_{h}^{\Gamma}$, then we simply consider $\llbracket q \rrbracket_{\sigma}=\{q\}_{\sigma}=\left.q\right|_{\sigma}$.

Moreover, let $\mathbb{P}_{n}(K)$ be the set of all real polynomials of degree less or equal than $n$ over $K$ and $h=\max _{K \in \mathcal{T}_{h}}\left(h_{K}\right)$ be the meshsize of $\mathcal{T}_{h}$. We define the following trial finite-dimensional subspaces for velocity $\boldsymbol{u}$ and pressure $p$ on a triangular mesh:

$$
\mathcal{V}_{h}:=\left\{\boldsymbol{v} \in \mathbf{L}^{2}(\Omega):\left.\boldsymbol{v}\right|_{K} \in \mathbb{P}_{1}(K)^{2} \forall K \in \mathcal{T}_{h}\right\}, \mathcal{Q}_{h}:=\left\{q \in L_{0}^{2}(\Omega):\left.q\right|_{K} \in \mathbb{P}_{0}(K) \forall K \in \mathcal{T}_{h}\right\} .
$$

For $\boldsymbol{v}_{h} \in \mathcal{V}_{h}$ we denote by $\llbracket \boldsymbol{v}_{h} \rrbracket_{\sigma}=\left.\boldsymbol{v}_{h}\right|_{K}-\left.\boldsymbol{v}_{h}\right|_{L}$ and $\left\{\boldsymbol{v}_{h}\right\}_{\sigma}=\frac{1}{2}\left(\left.\boldsymbol{v}_{h}\right|_{K}+\left.\boldsymbol{v}_{h}\right|_{L}\right)$ its jump and average across $\sigma$, respectively.

The test space for velocity on the dual mesh is defined as

$$
\mathcal{V}_{h}^{\sharp}:=\left\{\boldsymbol{v} \in \mathbf{L}^{2}(\Omega):\left.\boldsymbol{v}\right|_{D_{\sigma}} \in \mathbb{P}_{0}\left(D_{\sigma}\right)^{2} \forall D_{\sigma} \in \mathcal{T}_{h}^{\sharp}\right\} .
$$

After defining the space $\mathcal{V}(h):=\mathcal{V}_{h}+\left(\mathbf{H}^{2}(\Omega) \cap \mathbf{H}_{0}^{1}(\Omega)\right)$, we can connect $\mathcal{V}(h)$ to $\mathcal{V}_{h}^{\sharp}$ via the projection $\operatorname{map} \mathcal{P}^{\sharp}: \mathcal{V}_{h} \rightarrow \mathcal{V}_{h}^{\sharp}$ as follows:

$$
\left.\mathcal{P}^{\sharp} \boldsymbol{v}\right|_{D_{\sigma}}=\left.\frac{1}{h_{\sigma}} \int_{\sigma} \boldsymbol{v}\right|_{D_{\sigma}} \mathrm{d} s,
$$

and applying the Cauchy-Schwarz inequality, we obtain

$$
\llbracket \mathcal{P}^{\sharp} \boldsymbol{v}_{h} \rrbracket_{\sigma}^{2}=h_{\sigma}^{-2}\left(\int_{\sigma} \llbracket \boldsymbol{v}_{h} \rrbracket_{\sigma} \mathrm{d} s\right)^{2} \mathrm{~d} s \leq h_{\sigma}^{-2}\left(\int_{\sigma} \mathrm{d} s\right)\left(\int_{\sigma} \llbracket \boldsymbol{v}_{h} \rrbracket_{\sigma}^{2} \mathrm{~d} s\right)=h_{\sigma}^{-1} \int_{\sigma} \llbracket \boldsymbol{v}_{h} \rrbracket_{\sigma}^{2} \mathrm{~d} s .
$$

Moreover, we note that $\mathcal{P}^{\sharp}$ is self-adjoint with respect to the $L^{2}$-inner product, i.e.,

$$
\left(\mathcal{P}^{\sharp} \boldsymbol{u}_{h}, \boldsymbol{v}_{h}\right)=\left(\boldsymbol{u}_{h}, \mathcal{P}^{\sharp} \boldsymbol{v}_{h}\right) \quad \forall \boldsymbol{u}_{h}, \boldsymbol{v}_{h} \in \mathcal{V}_{h}
$$

A proof of the scalar version of $(3.2)$ is given in [8, p. 373]. In addition, if we define $\left\|\boldsymbol{v}_{h}\right\|_{0, \Omega}:=$ $\left(\boldsymbol{v}_{h}, \mathcal{P}^{\sharp} \boldsymbol{v}_{h}\right)_{\Omega}^{1 / 2}$, then $\|\cdot\|_{0, \Omega}$ and $\|\cdot\|_{0, \Omega}$ are equivalent (see [8, Lemma 3.5]), i.e., there exist positive constants $C_{1}$ and $C_{2}$ that are independent of $h$ such that

$$
C_{1}\left\|\boldsymbol{v}_{h}\right\|_{0, \Omega} \leq\left\|\boldsymbol{v}_{h}\right\|_{0, \Omega} \leq C_{2}\left\|\boldsymbol{v}_{h}\right\|_{0, \Omega} \quad \forall \boldsymbol{v}_{h} \in \mathcal{V}_{h}
$$

Also, further properties of $\mathcal{P}^{\sharp}$ follow from the definition of the diamond mesh (see proofs in e.g. [8, 9, 32]):

Lemma 3.1. Let $\boldsymbol{v}_{h} \in \mathcal{V}_{h}, K \in \mathcal{T}_{h}$ and $\sigma \subset \partial K$. Then the following properties hold:

$$
\begin{gathered}
\int_{K}\left(\boldsymbol{v}_{h}-\mathcal{P}^{\sharp} \boldsymbol{v}_{h}\right) \mathrm{d} s=0, \quad \int_{\sigma}\left(\boldsymbol{v}_{h}-\mathcal{P}^{\sharp} \boldsymbol{v}_{h}\right) \mathrm{d} s=0, \quad\left\|\mathcal{P}^{\sharp} \boldsymbol{v}_{h}\right\|_{0, \Omega}=\left\|\boldsymbol{v}_{h}\right\|_{0, \Omega}, \\
\left\|\boldsymbol{v}_{h}-\mathcal{P}^{\sharp} \boldsymbol{v}_{h}\right\|_{0, K} \leq C h_{K}\left|\boldsymbol{v}_{h}\right|_{1, K}, \quad \llbracket \boldsymbol{v}_{h} \rrbracket_{\sigma}=\mathbf{0} \Rightarrow \llbracket \mathcal{P}^{\sharp} \boldsymbol{v}_{h} \rrbracket_{\sigma}=\mathbf{0} .
\end{gathered}
$$

Multiplying 1.1b and 1.1c by $\boldsymbol{\xi}_{h} \in \mathcal{V}_{h}^{\sharp}$ and $q_{h} \in \mathcal{Q}_{h}$, respectively, using the Gauss divergence theorem on each dual element $D_{\sigma}$, and adding over all the dual elements, we arrive at

$$
\begin{array}{r}
\left(\mathbf{K}^{-1}(\boldsymbol{x}) \boldsymbol{u}, \boldsymbol{\xi}_{h}\right)_{\Omega}-\sum_{D_{\sigma} \in \mathcal{T}_{h}^{\sharp}} \int_{\partial D_{\sigma}} \mu(\phi) \boldsymbol{\varepsilon}(\boldsymbol{u}) \boldsymbol{n} \cdot \boldsymbol{\xi}_{h} \mathrm{~d} s+\sum_{D_{\sigma} \in \mathcal{T}_{h}^{\sharp}} \int_{\partial D_{\sigma}} p \boldsymbol{n} \cdot \boldsymbol{\xi}_{h} \mathrm{~d} s=\left(\phi \boldsymbol{g}, \boldsymbol{\xi}_{h}\right)_{\Omega} \quad \forall \boldsymbol{\xi}_{h} \in \mathcal{V}_{h}^{\sharp}, \\
\sum_{K \in \mathcal{T}_{h}} \int_{K} q_{h} \operatorname{div} \boldsymbol{u} \mathrm{d} \boldsymbol{x}=0 \quad \forall q_{h} \in \mathcal{Q}_{h} .
\end{array}
$$


Let $D_{\sigma_{j}} \in \mathcal{T}_{h}^{\sharp}(j=1,2,3)$ be the dual elements (sub-triangles) formed by barycentric subdivision of a generic element $K \in \mathcal{T}_{h}$, see Figure 1 (right). Then we can rewrite the second term of the above expression as

$$
\begin{aligned}
\sum_{D_{\sigma} \in \mathcal{T}_{h}^{\sharp}} \int_{\partial D_{\sigma}} \mu(\phi) \boldsymbol{\varepsilon}(\boldsymbol{u}) \boldsymbol{n} \cdot \boldsymbol{\xi}_{h} \mathrm{~d} s & =\sum_{K \in \mathcal{T}_{h}} \sum_{j=1}^{3} \int_{\partial D_{\sigma_{j}}} \mu(\phi) \boldsymbol{\varepsilon}(\boldsymbol{u}) \boldsymbol{n} \cdot \boldsymbol{\xi}_{h} \mathrm{~d} s \\
& =\sum_{K \in \mathcal{T}_{h}} \sum_{j=1}^{3} \int_{s_{j+1} b_{K} s_{j}} \mu(\phi) \boldsymbol{\varepsilon}(\boldsymbol{u}) \boldsymbol{n} \cdot \boldsymbol{\xi}_{h} \mathrm{~d} s+\sum_{K \in \mathcal{T}_{h}} \int_{\partial K} \mu(\phi) \boldsymbol{\varepsilon}(\boldsymbol{u}) \boldsymbol{n} \cdot \boldsymbol{\xi}_{h} \mathrm{~d} s
\end{aligned}
$$

where $s_{4}=s_{1}$. First we note that by using the definition of jump and average, a simple computation yields

$$
\sum_{K \in \mathcal{T}_{h}} \int_{\partial K} q \boldsymbol{v} \cdot \boldsymbol{n} \mathrm{d} s=\sum_{\sigma \in \mathcal{E}_{h}^{\text {int }}} \int_{\sigma} \llbracket q \boldsymbol{n} \rrbracket_{\sigma} \cdot\{\boldsymbol{v}\}_{\sigma} \mathrm{d} s+\sum_{\sigma \in \mathcal{E}_{h}} \int_{\sigma}\{q \boldsymbol{n}\}_{\sigma} \cdot \llbracket \boldsymbol{v} \rrbracket_{\sigma} \mathrm{d} s,
$$

where $\mathcal{E}_{h}^{\text {int }}:=\mathcal{E}_{h} \backslash \mathcal{E}_{h}^{\Gamma}$. Now we define the following forms:

$$
\begin{aligned}
c_{0}\left(\boldsymbol{u}, \xi_{\mathbf{h}} ; \phi\right) & :=-\sum_{K \in \mathcal{T}_{h}} \sum_{j=1}^{3} \int_{s_{j+1} b_{K} s_{j}} \mu(\phi) \boldsymbol{\varepsilon}(\boldsymbol{u}) \boldsymbol{n} \cdot \boldsymbol{\xi}_{h} \mathrm{~d} s-\sum_{\sigma \in \mathcal{E}_{h}} \int_{\sigma}\{\mu(\phi) \boldsymbol{\varepsilon}(\boldsymbol{u}) \boldsymbol{n}\}_{\sigma} \cdot \llbracket \boldsymbol{\xi}_{h} \rrbracket_{\sigma} \mathrm{d} s, \\
b_{0}\left(\boldsymbol{\xi}_{h}, p\right) & :=\sum_{K \in \mathcal{T}_{h}} \sum_{j=1}^{3} \int_{s_{j+1} b_{K} s_{j}} p \boldsymbol{n} \cdot \boldsymbol{\xi}_{h} \mathrm{~d} s+\sum_{\sigma \in \mathcal{E}_{h}} \int_{\sigma}\{p\}_{\sigma} \llbracket \boldsymbol{\xi}_{h} \rrbracket_{\sigma} \mathrm{d} s .
\end{aligned}
$$

Then, since the exact solutions $(\boldsymbol{u}, p)$ satisfy $\llbracket \varepsilon(\boldsymbol{u}) \boldsymbol{n} \rrbracket_{\sigma}=\mathbf{0}$ and $\llbracket p \boldsymbol{n} \rrbracket_{\sigma}=\mathbf{0}$ on every $\sigma \in \mathcal{E}_{h}^{\text {int }}$, it is easy to see that the solution $(\boldsymbol{u}, p)$ of $1.1 \mathrm{~b}, 11.1 \mathrm{c}$ satisfies the following problem (tested against discrete functions, as in classical DG methods; see also [46]):

$$
\begin{aligned}
a\left(\boldsymbol{u}, \boldsymbol{\xi}_{h}\right)+c_{0}\left(\boldsymbol{u}, \boldsymbol{\xi}_{h} ; \phi\right)+b_{0}\left(\boldsymbol{\xi}_{h}, p\right)-d\left(\phi, \boldsymbol{\xi}_{h}\right)=0 & \forall \boldsymbol{\xi}_{h} \in \mathcal{V}_{h}^{\sharp}, \\
b\left(\boldsymbol{u}, q_{h}\right)=0 & \forall q_{h} \in \mathcal{Q}_{h} .
\end{aligned}
$$

Now, using the definition of $\mathcal{P}^{\sharp}$ along with (3.6) and (3.7), we can define the following DFVE scheme for the Brinkman equations: Find $\boldsymbol{u}_{h} \in \mathcal{V}_{h}$ and $p_{h} \in \mathcal{Q}_{h}$ such that

$$
\begin{aligned}
a_{h}\left(\boldsymbol{u}_{h}, \boldsymbol{v}_{h}\right)+c_{h}\left(\boldsymbol{u}_{h}, \boldsymbol{v}_{h} ; \phi_{h}\right)+C_{h}\left(\boldsymbol{v}_{h}, p_{h}\right)-d_{h}\left(\phi_{h}, \boldsymbol{v}_{h}\right)=0 & \forall \boldsymbol{v}_{h} \in \mathcal{V}_{h}, \\
B_{h}\left(\boldsymbol{u}_{h}, q_{h}\right)=0 & \forall q_{h} \in \mathcal{Q}_{h},
\end{aligned}
$$

with

$$
\begin{aligned}
a_{h}\left(\boldsymbol{w}_{h}, \boldsymbol{v}_{h}\right):= & \left(\mathbf{K}^{-1}(\boldsymbol{x}) \boldsymbol{w}_{h}, \mathcal{P}^{\sharp} \boldsymbol{v}_{h}\right)_{\Omega}, \quad B_{h}\left(\boldsymbol{w}_{h}, q_{h}\right):=b\left(\boldsymbol{w}_{h}, q_{h}\right)-\sum_{\sigma \in \mathcal{E}_{h}} \int_{\sigma}\left\{q_{h}\right\}_{\sigma} \llbracket \boldsymbol{w}_{h} \rrbracket_{\sigma} \mathrm{d} s, \\
c_{h}\left(\boldsymbol{w}_{h}, \boldsymbol{v}_{h} ; \phi_{h}\right):= & -\sum_{K \in \mathcal{T}_{h}} \sum_{j=1}^{d+1} \int_{s_{j+1} b_{K} s_{j}} \mu\left(\phi_{h}\right) \varepsilon\left(\boldsymbol{w}_{h}\right) \boldsymbol{n} \cdot \mathcal{P}^{\sharp} \boldsymbol{v}_{h} \mathrm{~d} s-\sum_{\sigma \in \mathcal{E}_{h}} \int_{\sigma}\left\{\mu\left(\phi_{h}\right) \boldsymbol{\varepsilon}\left(\boldsymbol{w}_{h}\right) \boldsymbol{n}\right\}_{\sigma} \cdot \llbracket \mathcal{P}^{\sharp} \boldsymbol{v}_{h} \rrbracket_{\sigma} \mathrm{d} s \\
& -\sum_{\sigma \in \mathcal{E}_{h}} \int_{\sigma}\left\{\mu\left(\phi_{h}\right) \varepsilon\left(\boldsymbol{v}_{h}\right) \boldsymbol{n}\right\}_{\sigma} \cdot \llbracket \mathcal{P}^{\sharp} \boldsymbol{w}_{h} \rrbracket_{\sigma} \mathrm{d} s+\sum_{\sigma \in \mathcal{E}_{h}} \int_{\sigma} \frac{\alpha_{d}}{h_{\sigma}} \llbracket \boldsymbol{w}_{h} \rrbracket_{\sigma} \cdot \llbracket \boldsymbol{v}_{h} \rrbracket_{\sigma} \mathrm{d} s, \\
C_{h}\left(\boldsymbol{v}_{h}, r_{h}\right):= & \sum_{K \in \mathcal{T}_{h}} \sum_{j=1}^{d+1} \int_{s_{j+1} b_{K} s_{j}} r_{h} \boldsymbol{n} \cdot \mathcal{P}^{\sharp} \boldsymbol{v}_{h} \mathrm{~d} s+\sum_{\sigma \in \mathcal{E}_{h}} \int_{\sigma}\left\{r_{h}\right\}_{\sigma} \llbracket \boldsymbol{v}_{h} \rrbracket_{\sigma} \mathrm{d} s, \\
d_{h}\left(\phi_{h}, \boldsymbol{v}_{h}\right):= & \left(\phi_{h} \boldsymbol{g}, \mathcal{P}^{\sharp} \boldsymbol{v}_{h}\right)_{\Omega}
\end{aligned}
$$

for all $\boldsymbol{w}_{h}, \boldsymbol{v}_{h} \in \mathcal{V}_{h}$ and $r_{h}, q_{h} \in \mathcal{Q}_{h}$, and where $\alpha_{d}$ is the penalty parameter to be determined at a later stage. We note that since $\llbracket \boldsymbol{u} \rrbracket_{\sigma}$ and $\llbracket \mathcal{P}^{\sharp} \boldsymbol{u} \rrbracket_{\sigma}$ are zero (see $(3.5)$ ), the formulation (3.8), (3.9) is consistent 
with (3.6), 3.7). We stress that in the definition of $c_{h}$, we could have also added the third term (the one having a factor $\llbracket \mathcal{P}^{\sharp} \boldsymbol{w}_{h} \rrbracket_{\sigma}$ and which is essentially a zero term if we replace $\boldsymbol{w}_{h}$ by $\boldsymbol{u}$ ) instead of subtracting it from the formulation. In such a case, the coercivity (for a given $\phi_{h}$ ) of $c_{h}$ would not depend on the penalty parameter $\alpha_{d}$ (for more details, see [33); however, the derivation of $L^{2}$ error estimates is not straightforward, as one can not appeal to classical duality arguments. Furthermore, the proposed DFVE scheme 3.8, 3.9 is locally conservative, which is essential while seeking the numerical approximation of problems following certain laws of conservation.

Now using the definition of $a_{h}(\cdot, \cdot), \sqrt{1.4}$ and $(3.3)$, we see that there exists a positive constant $C$ independent of $h$ such that

$$
a_{h}\left(\boldsymbol{v}_{h}, \boldsymbol{v}_{h}\right) \geq C\left\|\boldsymbol{v}_{h}\right\|_{0, \Omega}^{2} .
$$

For our future analysis we also define the following natural mesh-dependent norms for all $\boldsymbol{v}_{h} \in \mathcal{V}(h)$ :

$$
\left\|\boldsymbol{v}_{h}\right\|_{h}^{2}:=\sum_{K \in \mathcal{T}_{h}}\left|\boldsymbol{v}_{h}\right|_{1, K}^{2}+\sum_{\sigma \in \mathcal{E}_{h}} h_{\sigma}^{-1}\left\|\llbracket \boldsymbol{v}_{h} \rrbracket_{\sigma}\right\|_{0, \sigma}^{2}, \quad\left\|\boldsymbol{v}_{h}\right\|_{1, h}^{2}:=\left\|\boldsymbol{v}_{h}\right\|_{h}^{2}+\sum_{K \in \mathcal{T}_{h}} h_{K}^{2}\left|\boldsymbol{v}_{h}\right|_{2, K}^{2}
$$

An application of the standard inverse inequality yields

$$
\left\|\boldsymbol{v}_{h}\right\|_{1, h} \leq C\left\|\boldsymbol{v}_{h}\right\|_{h} \quad \forall \boldsymbol{v}_{h} \in \mathcal{V}_{h}
$$

Hence, $\|\cdot\|_{h}$ and $\|\cdot\|_{1, h}$ are equivalent on $\mathcal{V}_{h}$. Moreover, by arguments similar to those used in the derivation of [46, Lemma 4.1], it is easy to show that the following inf-sup condition holds for our choice of $\mathcal{V}_{h}$ and $\mathcal{Q}_{h}$ :

$$
\sup _{\boldsymbol{v}_{h} \in \mathcal{V}_{h}} \frac{B_{h}\left(\boldsymbol{v}_{h}, q_{h}\right)}{\left\|\boldsymbol{v}_{h}\right\|_{h}} \geq \beta_{1}\left\|q_{h}\right\|_{0, \Omega}
$$

where $\beta_{1}>0$ is independent of $h$.

Next, we develop a relation between $\|\cdot\|$ and $\|\cdot\|_{h}$, to be used in deriving error estimates for velocity. For this, first we note the following relation (see [46]):

$$
\left\|\boldsymbol{v}_{h}\right\|_{0, \Omega}^{2} \leq C\left[\sum_{K \in \mathcal{T}_{h}}\left|\boldsymbol{v}_{h}\right|_{1, K}^{2}+\sum_{\sigma \in \mathcal{E}_{h}} \llbracket \mathcal{P}^{\sharp} \boldsymbol{v}_{h} \rrbracket_{\sigma}^{2}+\sum_{K \in \mathcal{T}_{h}} h_{K}^{2}\left|\boldsymbol{v}_{h}\right|_{2, K}^{2}\right] \quad \forall \boldsymbol{v}_{h} \in \mathcal{V}_{h} .
$$

Therefore, 3.1 and 3.12 imply the following discrete Poincaré-Friedrichs inequality:

$$
\left\|\boldsymbol{v}_{h}\right\|_{0, \Omega} \leq C\left\|\boldsymbol{v}_{h}\right\|_{h} \quad \forall \boldsymbol{v}_{h} \in \mathcal{V}_{h}
$$

where $C$ is independent of $h$. We also note that for a given $\phi_{h}$ and sufficiently large $\alpha_{d}$, the bilinear form $c_{h}\left(\cdot, \cdot ; \phi_{h}\right)$ will be coercive with respect to $\|\cdot\|_{h}$, see [33, Lemma 4], i.e., there exists a constant $\alpha$ independent of $h$ such that

$$
c_{h}\left(\boldsymbol{v}_{h}, \boldsymbol{v}_{h} ; \phi_{h}\right) \geq \alpha\left\|\boldsymbol{v}_{h}\right\|_{h}^{2} .
$$

An application of the Gauss divergence theorem and (3.1) provide the following relationship between the bilinear forms $B_{h}(\cdot, \cdot)$ and $C_{h}(\cdot, \cdot)$ (to be used for the error estimates for velocity and pressure; see [46]):

Lemma 3.2. For a given $\boldsymbol{v} \in \mathcal{V}(h)$, we have

$$
C_{h}\left(\boldsymbol{v}, q_{h}\right)=-B_{h}\left(\boldsymbol{v}, q_{h}\right) \quad \forall q_{h} \in \mathcal{Q}_{h} .
$$

Moreover, $C_{h}(\cdot, \cdot)$ is bounded in the following sense:

$$
\left|C_{h}(\boldsymbol{v}, q)\right| \leq C\|\boldsymbol{v}\|_{1, h}\left[\|q\|_{0, \Omega}+\left(\sum_{K} h_{K}^{2}|q|_{1, K}^{2}\right)^{1 / 2}\right] \quad \forall \boldsymbol{v} \in \mathcal{V}(h), \quad q \in L_{0}^{2}(\Omega) .
$$


Remark 3.1. It should be noticed that the constants involved in the stability analysis given above (for instance the coercivity constant in (3.15) may depend on $\mu$. In many circumstances related to Brinkman flows it is of interest to derive stability bounds which are independent of the viscosity, so they can be also valid in the Darcy limit $\mu \rightarrow 0$ (cf. [4, 5]). We stress that our analysis can be modified accordingly by redefining the mesh-dependent norms in a way suggested by the involved multilinear form. For example, the coercivity of $c_{h}$ can be established using the following norm

$$
\left\|\boldsymbol{v}_{h}\right\|_{h}^{2}=\mu_{\min } \sum_{K \in \mathcal{T}_{h}}\left|\boldsymbol{v}_{h}\right|_{1, K}^{2}+\sum_{\sigma \in \mathcal{E}_{h}} h_{\sigma}^{-1}\left\|\llbracket \boldsymbol{v}_{h} \rrbracket_{\sigma}\right\|_{0, \sigma}^{2},
$$

even if $\mu_{\mathrm{min}}=0$. If needed, other norms, weighted (involving e.g. $\mu_{\max }$ ) or not, can be used for deriving boundedness.

\subsection{Error estimates for velocity and pressure}

In order to derive estimates for $\boldsymbol{u}$ and $p$, we define the mapping $\pi_{K}: H^{1}(K) \longrightarrow P_{1}(K)$ by

$$
\int_{\sigma_{j}} \pi_{K} v \mathrm{~d} s=\int_{\sigma_{j}} v \mathrm{~d} s \quad \forall \boldsymbol{v} \in H^{1}(K), \quad j=1,2,3,
$$

where $\sigma_{j}, j=1,2,3$ are three edges of the triangle $K$. Also, the operator $\pi_{K}$ satisfies the following approximation properties (see [39])

$$
\left|\pi_{K} v-v\right|_{s, K} \leq C h^{2-s}|\boldsymbol{v}|_{2, K} \quad \forall v \in H^{2}(K), \quad s=0,1,2 .
$$

Then the projection map $\mathbf{I}_{h}: \mathbf{H}_{0}^{1}(\Omega) \longrightarrow \mathcal{V}_{h}$ defined by $\left.\left(\mathbf{I}_{h} \boldsymbol{v}\right)_{i}\right|_{K}=\pi_{K} v_{i} i=1,2$ satisfies (see [46]):

$$
B\left(\boldsymbol{u}-\mathbf{I}_{h} \boldsymbol{u}, q_{h}\right)=0 \quad \forall q_{h} \in \mathcal{Q}_{h} .
$$

Moreover, the definition of $\|\cdot\|_{h}$ and (3.18) imply that $\mathbf{I}_{h}$ satisfies:

$$
\begin{array}{r}
\left\|\boldsymbol{u}-\mathbf{I}_{h} \boldsymbol{u}\right\|_{h} \leq C h\|u\|_{2, \Omega}, \\
\left\|\boldsymbol{u}-\mathbf{I}_{h} \boldsymbol{u}\right\|_{0, \Omega} \leq C h^{2}\|u\|_{2, \Omega} .
\end{array}
$$

Also, let $\Pi_{h}$ be the $L^{2}$ projection from $L_{0}^{2}(\Omega)$ to $\mathcal{Q}_{h}$ with the following approximation property:

$$
\left\|p-\Pi_{h} p\right\|_{0, \Omega} \leq C h\|p\|_{1, \Omega} .
$$

Since the saturation $\phi$ appears in the pressure-velocity equation $1.1 \mathrm{~b}$, the following lemma shows that the error estimates of $p$ and $\boldsymbol{u}$ depend on the estimates of $\phi$.

Lemma 3.3. Let $(\boldsymbol{u}, p)$ and $\left(\boldsymbol{u}_{h}, p_{h}\right)$ be the solutions of $(3.6)$, (3.7) and (3.8), (3.9), respectively. Then there exists a constant $C$ independent of $h$, but which may depend on the bound of $\boldsymbol{u}$, such that

$$
\left\|\boldsymbol{u}-\boldsymbol{u}_{h}\right\|_{h}+\left\|p-p_{h}\right\|_{0, \Omega} \leq C\left[h\left(\|\boldsymbol{u}\|_{2, \Omega}+\|p\|_{1, \Omega}\right)+\left\|\phi-\phi_{h}\right\|_{0, \Omega}+h\left(\sum_{K}\left\|\nabla\left(\phi-\phi_{h}\right)\right\|_{0, K}^{2}\right)^{1 / 2}\right] .
$$

Proof. We take $\boldsymbol{u}-\boldsymbol{u}_{h}=\boldsymbol{\eta}+\boldsymbol{\eta}_{h}$ and $p-p_{h}=\xi+\xi_{h}$, where $\boldsymbol{\eta}:=\boldsymbol{u}-\mathbf{I}_{h} \boldsymbol{u}, \boldsymbol{\eta}_{h}:=\mathbf{I}_{h} \boldsymbol{u}-\boldsymbol{u}_{h}, \xi:=p-\Pi_{h} p$ and $\xi_{h}:=\Pi_{h} p-p_{h}$. Since estimates for $\boldsymbol{\eta}$ and $\xi$ are known, it is enough to find the estimates of $\boldsymbol{\eta}_{h}$ and $\xi_{h}$.

First we note that from $3.6-(3.7)$, the pair $(\boldsymbol{u}, p)$ satisfies

$$
a_{h}\left(\boldsymbol{u}, \boldsymbol{v}_{h}\right)+c_{h}\left(\boldsymbol{u}, \boldsymbol{v}_{h} ; \phi\right)+C_{h}\left(\boldsymbol{v}_{h}, p\right)=d_{h}\left(\phi, \boldsymbol{v}_{h}\right) \quad \forall \boldsymbol{v}_{h} \in \mathcal{V}_{h}
$$




$$
B_{h}\left(\boldsymbol{u}, q_{h}\right)=0 \quad \forall q_{h} \in \mathcal{Q}_{h} .
$$

Subtracting (3.23) from (3.8) and (3.24) from (3.9), respectively, and using (3.19), we arrive at

$$
\begin{gathered}
a_{h}\left(\boldsymbol{\eta}_{h}, \boldsymbol{v}_{h}\right)+c_{h}\left(\boldsymbol{\eta}_{h}, \boldsymbol{v}_{h} ; \phi_{h}\right)+C_{h}\left(\boldsymbol{v}_{h}, \xi_{h}\right)= \\
d_{h}\left(\phi, \boldsymbol{v}_{h}\right)-d_{h}\left(\phi_{h}, \boldsymbol{v}_{h}\right)-C_{h}\left(\boldsymbol{v}_{h}, \xi\right)-a_{h}\left(\boldsymbol{\eta}, \boldsymbol{v}_{h}\right) \\
-\left(c_{h}\left(\boldsymbol{u}, \boldsymbol{v}_{h} ; \phi\right)-c_{h}\left(\boldsymbol{u}, \boldsymbol{v}_{h} ; \phi_{h}\right)\right) \quad \forall \boldsymbol{v}_{h} \in \mathcal{V}_{h}, \\
B_{h}\left(\eta_{h}, q_{h}\right)=0 \quad \forall q_{h} \in \mathcal{Q}_{h} .
\end{gathered}
$$

Next, choosing $\boldsymbol{v}_{h}=\boldsymbol{\eta}_{h}$ and employing (3.16), we deduce that $C_{h}\left(\boldsymbol{\eta}_{h}, \xi_{h}\right)=0$. Therefore, as a consequence of 3.10 and 3.15 , we obtain from 3.25

$$
\left\|\boldsymbol{\eta}_{h}\right\|_{h}^{2} \leq C\left[\left(d_{h}\left(\phi, \boldsymbol{\eta}_{h}\right)-d_{h}\left(\phi_{h}, \eta_{h}\right)\right)-a_{h}\left(\boldsymbol{\eta}, \boldsymbol{\eta}_{h}\right)-\left(c_{h}\left(\boldsymbol{u}, \boldsymbol{\eta}_{h} ; \phi\right)-c_{h}\left(\boldsymbol{u}, \boldsymbol{\eta}_{h} ; \phi_{h}\right)\right)-C_{h}\left(\boldsymbol{\eta}_{h}, \xi\right)\right]
$$

Note that the definition of $d_{h}(\cdot, \cdot)$, the Cauchy-Schwarz inequality, and $(3.4),(3.14)$, immediately yield

$$
\left|d_{h}\left(\phi, \boldsymbol{\eta}_{h}\right)-d_{h}\left(\phi_{h}, \boldsymbol{\eta}_{h}\right)\right| \leq C\left\|\phi-\phi_{h}\right\|_{0, \Omega}\left\|\boldsymbol{\eta}_{h}\right\|_{h} .
$$

Again, an application of Cauchy-Schwarz inequality together with (3.4), (3.14) and (3.21) allows us to write

$$
\left|a_{h}\left(\boldsymbol{\eta}, \boldsymbol{\eta}_{h}\right)\right| \leq\|\boldsymbol{\eta}\|_{0, \Omega}\left\|\boldsymbol{\eta}_{h}\right\|_{h} \leq C h^{2}\|\boldsymbol{u}\|_{2, \Omega}\left\|\boldsymbol{\eta}_{h}\right\|_{h} .
$$

In light of the assumptions on the viscosity given in $(1.3)$ and using the same arguments as used in the derivation of [12, Lemma 4.3], the following bound can be easily obtained:

$$
\left|c_{h}\left(\boldsymbol{u}, \boldsymbol{\eta}_{h} ; \phi\right)-c_{h}\left(\boldsymbol{u}, \boldsymbol{\eta}_{h} ; \phi_{h}\right)\right| \leq C\left[\left\|\phi-\phi_{h}\right\|_{0, \Omega}+h\left(\sum_{K \in \mathcal{T}_{h}}\left\|\nabla\left(\phi-\phi_{h}\right)\right\|_{0, K}^{2}\right)^{1 / 2}\right]\left\|\boldsymbol{\eta}_{h}\right\|_{h},
$$

and the following bound directly follows from $(3.17),(3.12)$ and by the definition of $\Pi_{h}$ :

$$
\left|C_{h}\left(\boldsymbol{\eta}_{h}, \xi\right)\right| \leq C h\left\|\boldsymbol{\eta}_{h}\right\|_{h}\|p\|_{1, \Omega}
$$

Combining all the bounds derived in $(3.26)-(3.27)$, we obtain from $(3.26)$ :

$$
\left\|\boldsymbol{\eta}_{h}\right\|_{h} \leq C\left[\left\|\phi-\phi_{h}\right\|_{0, \Omega}+h\left(\sum_{K \in \mathcal{T}_{h}}\left\|\nabla\left(\phi-\phi_{h}\right)\right\|_{0, K}^{2}\right)^{1 / 2}+h\left(\|\boldsymbol{u}\|_{2, \Omega}+\|p\|_{1, \Omega}\right)\right] .
$$

For estimating $\xi_{h}$ we proceed as follows: We choose $\boldsymbol{v}_{h}=\eta_{h}$ in (3.25), use (3.10), (3.15) and bounds obtained in (3.26)-(3.27), to arrive at

$$
C_{h}\left(\boldsymbol{\eta}_{h}, \xi_{h}\right) \leq C\left[\left\|\phi-\phi_{h}\right\|_{0, \Omega}+h\left(\sum_{K \in \mathcal{T}_{h}}\left\|\nabla\left(\phi-\phi_{h}\right)\right\|_{0, K}^{2}\right)^{1 / 2}+h\left(\|\boldsymbol{u}\|_{2, \Omega}+\|p\|_{1, \Omega}\right)\right]\left\|\boldsymbol{\eta}_{h}\right\|_{h}
$$

Now, an application of inf-sup condition (3.13) together with 3.16 yields

$$
\left\|\xi_{h}\right\|_{0, \Omega} \leq C\left[\left\|\phi-\phi_{h}\right\|_{0, \Omega}+h\left(\sum_{K \in \mathcal{T}_{h}}\left\|\nabla\left(\phi-\phi_{h}\right)\right\|_{0, K}^{2}\right)^{1 / 2}+h\left(\|\boldsymbol{u}\|_{2, \Omega}+\|p\|_{1, \Omega}\right)\right] .
$$

Using 3.20 and 3.22 , we complete the proof.

Remark 3.2. In the present setting, the convergence analysis for the approximate saturation $\phi_{h}$ remains an open problem. However, this quantity is uniformly bounded, as we show in Section 4. Together with these stability results and the regularity assumption on saturation, Lemma 3.3 suggests that a fixed-point iteration between the approximation of the Brinkman and the transport problems will be applied on discrete quantities whose discretization error remains uniformly bounded. 


\section{3. $D G$ discretization for the saturation equation}

We assume that each element $K$ can be mapped to a fixed reference element $\hat{K}$ using the operator $T_{K}: \hat{K} \rightarrow K$. In the special case of triangular elements, we will consider the space

$$
S_{h}^{k}:=\left\{s_{h}:\left.s_{h}\right|_{K} \in \mathbb{P}_{k}(K) \quad \forall K \in \mathcal{T}_{h}\right\},
$$

where $\mathbb{P}_{k}(K)$ denotes the space spanned by polynomials of degree at most $k$, defined on the element $K$.

Next, let us suppose that the approximate velocity field $\boldsymbol{u}_{h}$ has been computed previously on the primal mesh. We proceed to multiply the first equation in (1.1) by $s_{h} \in S_{h}^{k}$, integrate by parts over $K \in \mathcal{T}_{h}$, and replace the exact solution $\phi$ by its approximation $\phi_{h} \in S_{h}^{k}$ to obtain

$$
\begin{aligned}
& \frac{\mathrm{d}}{\mathrm{d} t}\left(\phi_{h}(\boldsymbol{x}, t), s_{h}(\boldsymbol{x})\right)_{K}-\left(\mathbf{F}\left(\phi_{h}(\boldsymbol{x}, t), \boldsymbol{u}_{h}, \boldsymbol{x}\right), \nabla s_{h}(\boldsymbol{x})\right)_{K} \\
& +\sum_{\sigma \in \partial K}\left\langle\mathbf{F}\left(\phi_{h}(\boldsymbol{x}, t), \boldsymbol{u}_{h}, \boldsymbol{x}\right) \cdot \boldsymbol{n}_{K, \sigma}, s_{h}(\boldsymbol{x})\right\rangle_{\sigma}=0 \quad \forall s_{h} \in S_{h}^{k},
\end{aligned}
$$

where $\boldsymbol{n}_{\sigma}$ is the unit outward normal to the edge $\sigma$. The term $\mathbf{F}\left(\phi_{h}(\boldsymbol{x}, t), \boldsymbol{u}_{h}, \boldsymbol{x}\right) \cdot \boldsymbol{n}_{K, \sigma}$ has to be specified appropriately, since $\phi_{h}(\boldsymbol{x}, t)$ is not necessarily continuous across the element boundary. Here, this flux function is approximated following [18, that is, we employ

$$
\mathbf{F}\left(\phi_{h}(\boldsymbol{x}, t), \boldsymbol{u}_{h}, \boldsymbol{x}\right) \cdot \boldsymbol{n}_{K, \sigma} \approx J\left(\phi_{h}\left(\boldsymbol{x}^{\mathrm{int}(K)}, t\right), \phi_{h}\left(\boldsymbol{x}^{\operatorname{ext}(K)}, t\right), \boldsymbol{u}_{h}, \boldsymbol{x}, \boldsymbol{n}_{\sigma}\right),
$$

and therefore 3.28 now reads

$$
\begin{aligned}
& \frac{\mathrm{d}}{\mathrm{d} t}\left(\phi_{h}(\boldsymbol{x}, t), s_{h}(\boldsymbol{x})\right)_{K}-\left(\mathbf{F}\left(\phi_{h}(\boldsymbol{x}, t), \boldsymbol{u}_{h}, \boldsymbol{x}\right), \nabla s_{h}(\boldsymbol{x})\right)_{K} \\
& +\sum_{\sigma \in \partial K}\left\langle J\left(\phi_{h}\left(\boldsymbol{x}^{\operatorname{int}(K)}, t\right), \phi_{h}\left(\boldsymbol{x}^{\operatorname{ext}(K)}, t\right), \boldsymbol{u}_{h}, \boldsymbol{x}, \boldsymbol{n}_{\sigma}\right), s_{h}(\boldsymbol{x})\right\rangle_{\sigma}=0 \quad \forall s_{h} \in S_{h}^{k} .
\end{aligned}
$$

The volume and surface integrals involved in the inner products are computed employing the following quadrature rules, where the nodes $x_{K_{j}} \in K$ and corresponding weights $\bar{\omega}_{j}, j=1, \ldots, M$, and the nodes $x_{i, \sigma} \in \sigma$ and corresponding weights $\omega_{i}, i=1, \ldots, N$, are specified later:

$$
\begin{aligned}
& \left(\mathbf{F}\left(\phi_{h}(\boldsymbol{x}, t), \boldsymbol{u}_{h}, \boldsymbol{x}\right), \nabla s_{h}(\boldsymbol{x})\right)_{K} \approx \sum_{j=1}^{M} \bar{\omega}_{j} \mathbf{F}\left(\phi_{h}\left(\boldsymbol{x}_{K_{j}}, t\right), \boldsymbol{u}_{h}\left(\boldsymbol{x}_{K_{j}}\right), \boldsymbol{x}_{K_{j}}\right) \cdot \nabla s_{h}\left(\boldsymbol{x}_{K_{j}}\right)|K|, \\
& \left\langle J\left(\phi_{h}\left(\boldsymbol{x}^{\operatorname{int}(K)}, t\right), \phi_{h}\left(\boldsymbol{x}^{\operatorname{ext}(K)}, t\right), \boldsymbol{u}_{h}, \boldsymbol{x}, \boldsymbol{n}_{\sigma}\right), s_{h}(\boldsymbol{x})\right\rangle_{\sigma} \\
& \approx \sum_{i=1}^{N} \omega_{i} J\left(\phi_{h}\left(\boldsymbol{x}_{i, \sigma}^{\operatorname{int}(K)}, t\right), \phi_{h}\left(\boldsymbol{x}_{i, \sigma}^{\operatorname{ext}(K)}, t\right), \boldsymbol{u}_{h}, \boldsymbol{x}_{i, \sigma}, \boldsymbol{n}_{\sigma}\right) s_{h}\left(\boldsymbol{x}_{i, \sigma}\right)|\sigma|,
\end{aligned}
$$

The corresponding weak formulation 3.29 is then replaced by

$$
\begin{aligned}
& \frac{\mathrm{d}}{\mathrm{d} t}\left(\phi_{h}(\boldsymbol{x}, t), s_{h}(\boldsymbol{x})\right)_{K}-\sum_{j=1}^{M} \bar{\omega}_{j} \mathbf{F}\left(\phi_{h}\left(\boldsymbol{x}_{K_{j}}, t\right), \boldsymbol{u}_{h}\left(\boldsymbol{x}_{K_{j}}\right), \boldsymbol{x}_{K_{j}}\right) \cdot \nabla s_{h}\left(\boldsymbol{x}_{K_{j}}\right)|K| \\
& +\sum_{\sigma \in \partial K} \sum_{i=1}^{N} \omega_{i} J\left(\phi_{h}\left(\boldsymbol{x}_{i, \sigma}^{\operatorname{int}(K)}, t\right), \phi_{h}\left(\boldsymbol{x}_{i, \sigma}^{\operatorname{ext}(K)}, t\right), \boldsymbol{u}_{h}, \boldsymbol{x}_{i, \sigma}, \boldsymbol{n}_{\sigma}\right) s_{h}\left(\boldsymbol{x}_{i, \sigma}\right)|\sigma|=0 \quad \forall s_{h} \in S_{h}^{k} .
\end{aligned}
$$

\subsection{Fully discrete $D G$ formulation}

We now choose a basis $\left\{\psi_{1}, \ldots \psi_{L}\right\}$ for the space $S_{h}^{k}$ and write the approximation of a given function $\phi_{h}$ in such a space as

$$
\phi_{h}(\boldsymbol{x}, t) \approx \sum_{j=1}^{L} \phi_{j}^{K}(t) \psi_{j}(\boldsymbol{x})
$$


Replacing $\phi_{h}$ in 3.30 by the right-hand side of (3.31) and choosing $s_{h}=\psi_{k}$ for $k=1, \ldots, L$, one may reduce the DG formulation to the following system of ODEs:

$$
\frac{\mathrm{d}}{\mathrm{d} t} \boldsymbol{\phi}_{h}^{K}(t)=-\mathbf{A}^{-1}(\boldsymbol{b}+\boldsymbol{c}), \quad \phi_{h}^{K}(t):=\left(\phi_{1}^{K}(t), \ldots, \phi_{L}^{K}(t)\right)^{\mathrm{T}},
$$

where $\mathbf{A}=\left(a_{j k}\right)_{L \times L}, \boldsymbol{b}=\left(b_{1}, \ldots, b_{L}\right)^{\mathrm{T}}$, and $\boldsymbol{c}=\left(c_{1}, \ldots, c_{L}\right)^{\mathrm{T}}$ with

$$
\begin{aligned}
& a_{j k}=\left(\psi_{j}(\boldsymbol{x}), \psi_{k}(\boldsymbol{x})\right)_{K}, \quad b_{k}=-\sum_{j=1}^{M} \bar{\omega}_{j} \mathbf{F}\left(\phi_{h}\left(\boldsymbol{x}_{K_{j}}, t\right), \boldsymbol{u}_{h}\left(\boldsymbol{x}_{K_{j}}\right)\right) \cdot \nabla \psi_{k}\left(\boldsymbol{x}_{K_{j}}\right)|K|, \\
& c_{k}=\sum_{\sigma \in \partial K} \sum_{i=1}^{N} \omega_{i} J\left(\phi_{h}\left(\boldsymbol{x}_{i, \sigma}^{\operatorname{int}(K)}, t\right), \phi_{h}\left(\boldsymbol{x}_{i, \sigma}^{\operatorname{ext}(K)}, t\right), \boldsymbol{u}_{h}, \boldsymbol{x}_{i, \sigma}, \boldsymbol{n}_{\sigma}\right) \psi_{k}\left(\boldsymbol{x}_{i, \sigma}\right)|\sigma|, \quad j, k=1, \ldots, L .
\end{aligned}
$$

We apply the second-order strong stability preserving (SSP) Runge-Kutta high order time discretization 38] (see also [26, 27]) in the time variable from $t^{n}$ to $t^{n+1}$ and therefore the local approximation for $\phi$ is computed from

$$
\phi_{h}\left(\boldsymbol{x}, t^{n+1}\right)=\left(\psi_{1}(\boldsymbol{x}), \ldots, \psi_{L}(\boldsymbol{x})\right) \phi_{h}^{K}\left(t^{n+1}\right) \quad \forall \boldsymbol{x} \in K .
$$

\subsection{Definition of the numerical flux}

To motivate the definition of the numerical flux, we assume for the moment that the flux vector $\mathbf{F}$ does not depend on $\boldsymbol{x}$. We write $\mathbf{F}=\left(F_{1}, F_{2}\right), \boldsymbol{n}=\left(n_{1}, n_{2}\right)$ and assume that both flux components $F_{1}$ and $F_{2}$ are functions of saturation $\phi$ only and are convex. We define the sign function

$$
\operatorname{sgn}(x)= \begin{cases}-1 & \text { if } x<0 \\ 0 & \text { if } x=0 \\ 1 & \text { if } x>0\end{cases}
$$

and we define

$$
\tilde{h}(\alpha, \beta, \boldsymbol{n}):=\hat{F}_{1}\left(\alpha, \beta, n_{1}\right)+\hat{F}_{2}\left(\alpha, \beta, n_{2}\right) .
$$

Depending on the normal directions, the components $\hat{F}_{1}$ and $\hat{F}_{2}$ of the numerical fluxes can be computed as follows:

$$
\hat{F}_{i}\left(\alpha, \beta, n_{i}\right)=\operatorname{sgn}\left(n_{i}\right)\left[\operatorname{sgn}\left(n_{i}\right) F_{i} n_{i}\left(\operatorname{sgn}\left(n_{i}\right) \delta_{i}^{1}\right) \vee \operatorname{sgn}\left(n_{i}\right) F_{i} n_{i}\left(\operatorname{sgn}\left(n_{i}\right) \delta_{i}^{2}\right)\right], \quad i=1,2 .
$$

Here we use the notation $F_{i} n_{i}(\cdot)=F_{i}(\cdot) n_{i}, i=1,2$ and $a \vee b=\max \{a, b\}$ and $a \wedge b=\min \{a, b\}$ for all $a, b \in \mathbb{R}$, set $\theta_{F_{i} n_{i}}$ such that

$$
F_{i} n_{i}\left(\theta_{F_{i} n_{i}}\right)=\min _{\phi \in[0,1]}\left(\operatorname{sgn}\left(n_{i}\right) F_{i} n_{i}(\phi)\right),
$$

and define $\delta_{i}^{1}:=\operatorname{sgn}\left(n_{i}\right) \alpha \vee \operatorname{sgn}\left(n_{i}\right) \theta_{F_{i} n_{i}}$ and $\delta_{i}^{2}:=\operatorname{sgn}\left(n_{i}\right) \beta \wedge \operatorname{sgn}\left(n_{i}\right) \theta_{F_{i} n_{i}}$.

Lemma 3.4. The numerical flux $\tilde{J}$ defined by 3.32, 3.33 satisfies the following essential properties.

(i) It is consistent, that is, $\tilde{J}(\phi, \phi, \boldsymbol{n})=\mathbf{F}(\phi) \cdot \boldsymbol{n}$ for all $\phi$.

(ii) It is monotone, that is, for given $\boldsymbol{n}, \tilde{J}(\alpha, \beta, \boldsymbol{n})$ is a non-decreasing function of $\alpha$ and a nonincreasing function of $\beta$.

(iii) It is conservative, i.e., $\tilde{J}(\alpha, \beta, \boldsymbol{n})=-\tilde{J}(\beta, \alpha,-\boldsymbol{n})$. 
Proof. For the consistency, let us concentrate on the case $n_{1}>0$ and $n_{2}<0$. It holds that

$$
\begin{aligned}
& \tilde{J}(\beta, \alpha,-\boldsymbol{n}) \\
& =\hat{F}_{1}\left(\beta, \alpha,-n_{1}\right)+\hat{F}_{2}\left(\beta, \alpha,-n_{2}\right) \\
& =\left(-F_{1} n_{1}\left(\beta \wedge \theta_{-F_{1} n_{1}}\right) \wedge-F_{1} n_{1}\left(\alpha \vee \theta_{-F_{1} n_{1}}\right)\right)+\left(-F_{2} n_{2}\left(\beta \vee \theta_{-F_{2} n_{2}}\right) \vee-F_{2} n_{2}\left(\alpha \wedge \theta_{-F_{2} n_{2}}\right)\right) \\
& =-\left(F_{1} n_{1}\left(\beta \wedge \theta_{-F_{1} n_{1}}\right) \vee F_{1} n_{1}\left(\alpha \vee \theta_{-F_{1} n_{1}}\right)\right)-\left(F_{2} n_{2}\left(\beta \vee \theta_{-F_{2} n_{2}}\right) \wedge F_{2} n_{2}\left(\alpha \wedge \theta_{-F_{2} n_{2}}\right)\right) \\
& =-\left(F_{1} n_{1}\left(\alpha \vee \theta_{-F_{1} n_{1}}\right) \vee F_{1} n_{1}\left(\beta \wedge \theta_{-F_{1} n_{1}}\right)\right)-\left(F_{2} n_{2}\left(\alpha \wedge \theta_{-F_{2} n_{2}}\right) \wedge F_{2} n_{2}\left(\beta \vee \theta_{-F_{2} n_{2}}\right)\right) \\
& =-\hat{F}_{1}\left(\alpha, \beta, n_{1}\right)-\hat{F}_{2}\left(\alpha, \beta, n_{2}\right)=-\tilde{J}(\alpha, \beta, \boldsymbol{n}) .
\end{aligned}
$$

The remaining properties can readily be established.

Since the flux vector $\mathbf{F}$ as given by 1.2 is in general discontinuous in $\boldsymbol{x}$, we apply the modified Godunov flux reconstruction proposed in [1]. The DFLU numerical flux is defined as

$$
J\left(\alpha, \beta, \boldsymbol{u}_{h}(\boldsymbol{x}), \boldsymbol{x}, \boldsymbol{n}\right)=\hat{F}_{1}\left(\alpha, \beta, n_{1}\right)+\hat{F}_{2}\left(\alpha, \beta, n_{2}\right),
$$

where the numerical flux components $\hat{F}_{1}$ and $\hat{F}_{2}$ are now computed as follows, where we use the notation $F_{i}^{-} n_{i}(\cdot)=F_{i}\left(\cdot, \boldsymbol{u}_{h}(\boldsymbol{x}), \boldsymbol{x}^{-}\right) n_{i}$ and $F_{i}^{+} n_{i}(\cdot)=F_{i}\left(\cdot, \boldsymbol{u}_{h}(\boldsymbol{x}), \boldsymbol{x}^{+}\right) n_{i}$. Since these components $F_{i}^{-} n_{i}$ and $F_{i}^{+} n_{i}$ follow the hypotheses $\left(H_{1}\right)$ and $\left(H_{2}\right)$ of [1], the corresponding expression for the numerical fluxes is given by

$$
\hat{F}_{i}\left(\alpha, \beta, n_{i}\right)=\operatorname{sgn}\left(n_{i}\right)\left[\operatorname{sgn}\left(n_{i}\right) F_{i}^{-} n_{i}\left(\operatorname{sgn}\left(n_{i}\right) \delta_{i}^{1}\right) \vee \operatorname{sgn}\left(n_{i}\right) F_{i}^{+} n_{i}\left(\operatorname{sgn}\left(n_{i}\right) \delta_{i}^{2}\right)\right], \quad i=1,2,
$$

where $\delta_{i}^{1}=\operatorname{sgn}\left(n_{i}\right) \alpha \vee \operatorname{sgn}\left(n_{i}\right) \theta_{F_{i}^{-} n_{i}}$ and $\delta_{i}^{2}=\operatorname{sgn}\left(n_{i}\right) \beta \wedge \operatorname{sgn}\left(n_{i}\right) \theta_{F_{i}^{+} n_{i}}$ and $\theta_{F_{i}^{ \pm} n_{i}}$ are such that

$$
F_{i}^{ \pm} n_{i}\left(\theta_{F_{i}^{ \pm} n_{i}}\right)=\min _{\phi \in[0,1]}\left(\operatorname{sgn}\left(n_{i}\right) F_{i}^{ \pm} n_{i}(\phi)\right) .
$$

Corollary 3.1. For fixed values of $\boldsymbol{u}_{h}(\boldsymbol{x}), \boldsymbol{x}$ and $\boldsymbol{n}$, the numerical flux J defined by (3.34), (3.35) has the following essential properties.

(i) It is monotone, that is, for given $\boldsymbol{n}, J\left(\alpha, \beta, \boldsymbol{u}_{h}(\boldsymbol{x}), \boldsymbol{x}, \boldsymbol{n}\right)$ is a non-decreasing function of $\alpha$ and a non-increasing function of $\beta$.

(ii) It is conservative, i.e., $J\left(\alpha, \beta, \boldsymbol{u}_{h}(\boldsymbol{x}), \boldsymbol{x}, \boldsymbol{n}\right)=-J\left(\beta, \alpha, \boldsymbol{u}_{h}(\boldsymbol{x}), \boldsymbol{x},-\boldsymbol{n}\right)$.

Note that the flux $h$ defined by (3.34, 3.35 is discontinuous in the spatial variable, and therefore the numerical flux is not consistent in the usual sense, but this does not compromise the possibility of obtaining the convergence results as we are able to show the stability properties (see [1, p. 181]).

\subsection{Limiter for triangular elements}

Plain high-order DG formulations $(k \geq 1)$ typically lack general stability properties exhibited by loworder methods, and spurious oscillations may develop. However, this issue can be resolved, for instance, by applying a limiter to the new solution at time $t^{n+1}$ :

$$
\phi_{h}^{n+1}=\Lambda \Pi_{h}\left(\phi_{h}\left(\boldsymbol{x}, t^{n+1}\right)\right),
$$

where the basic requirement is that the limiting operator $\Lambda \Pi_{h}$ should not change the cell average value, and should not affect the accuracy of the overall scheme in smooth regions. The many possibilities found in the literature (see e.g., [10, 15, 29, 34, 43]) suggest that the tuning of parameters (which strongly depend on the particular problem at hand) is often needed. Here we opt for the classical approach of [19], where for a piecewise linear function $\phi_{h}$, the limiter possesses the following properties 
1. Accuracy: if $\phi_{h}$ is a linear function, then $\Lambda \Pi_{h} \phi_{h}=\phi_{h}$.

2. Conservation of mass: for every $K \in \mathcal{T}_{h}$, we have $\int_{K} \Lambda \Pi_{h} \phi_{h} \mathrm{~d} \boldsymbol{x}=\int_{K} \phi_{h} \mathrm{~d} \boldsymbol{x}$.

3. Slope limiting: on each $K \in \mathcal{T}_{h}$ the gradient of $\Lambda \Pi_{h} \phi_{h}$ is not larger than that of $\phi_{h}$.

Let us consider the triangulation in Figure 2, where $b_{i}$ denotes the barycenter of the corresponding triangle $K_{i}$ for $i=0,1,2,3$ and $m_{1}$ is the midpoint of the edge face joining the triangle $K_{0}$ and $K_{1}$. For some $\alpha_{1}, \alpha_{2} \geq 0$, we can write $m_{1}-b_{0}=\alpha_{2}\left(b_{1}-b_{0}\right)+\alpha_{2}\left(b_{2}-b_{0}\right)$.

For any linear function $\phi_{h}$ we have the following identities:

$$
\phi_{h}\left(m_{1}\right)-\phi_{h}\left(b_{0}\right)=\alpha_{1}\left(\phi_{h}\left(b_{1}\right)-\phi_{h}\left(b_{0}\right)\right)+\alpha_{2}\left(\phi_{h}\left(b_{2}\right)-\phi_{h}\left(b_{0}\right)\right)
$$

and

Thus, we can write

$$
\bar{\phi}_{K_{i}}=\frac{1}{K_{i}} \int_{K_{i}} \phi_{h} \mathrm{~d} \boldsymbol{x}=\phi_{h}\left(b_{i}\right), \quad i=0,1,2,3
$$

$$
\tilde{\phi}_{h}\left(m_{1}, K_{0}\right) \equiv \phi_{h}\left(m_{1}\right)-\bar{\phi}_{K_{0}}=\alpha_{1}\left(\bar{\phi}_{K_{1}}-\bar{\phi}_{K_{0}}\right)+\alpha_{2}\left(\bar{\phi}_{K_{2}}-\bar{\phi}_{K_{0}}\right) \equiv \Delta \bar{\phi}\left(m_{1}, K_{0}\right) .
$$

Now if we choose a nodal Lagrangian basis $\left\{\psi_{1}, \psi_{2}, \psi_{3}\right\}$ for each element $K$ such that each basis function takes the value one at one of the midpoints of the edge faces and zero at the remaining midpoints, then for any $\boldsymbol{x} \in K_{0}$ we can write

$$
\phi_{h}(\boldsymbol{x})=\sum_{i=1}^{3} \phi_{h}\left(m_{i}\right) \psi_{i}(\boldsymbol{x})=\bar{\phi}_{K_{0}}+\sum_{i=1}^{3} \tilde{\phi}_{h}\left(m_{i}, K_{0}\right) \psi_{i}(\boldsymbol{x}),
$$

where $m_{1}, m_{2}, m_{3}$ are midpoints of the edges of the triangle $K_{0}$. To compute $\Lambda \Pi_{h}$, we first obtain the quantities

$$
\left.\Delta_{i}=\bar{m}\left\{\tilde{\phi}_{h}\left(m_{i}, K_{0}\right), \nu \Delta \bar{\phi}\left(m_{i}, K_{0}\right)\right)\right\},
$$

where $\nu>1$ and $\bar{m}$ is the modified min-mod function defined as follows:

$$
\bar{m}\left(a_{1}, a_{2}, \ldots, a_{m}\right)= \begin{cases}a_{1} & \text { if }\left|a_{1}\right| \leq \hat{M} h^{2} \\ \operatorname{minmod}\left(a_{1}, a_{2}, \ldots, a_{m}\right) & \text { otherwise }\end{cases}
$$

with the min-mod function is defined by

$$
\operatorname{minmod}\left(a_{1}, a_{2}, \ldots, a_{m}\right)= \begin{cases}s \min \left\{\left|a_{1}\right|, \ldots,\left|a_{m}\right|\right\} & \text { if } \operatorname{sgn}\left(a_{1}\right)=\operatorname{sgn}\left(a_{2}\right)=\cdots=\operatorname{sgn}\left(a_{m}\right)=: s, \\ 0 & \text { otherwise }\end{cases}
$$

where $\hat{M}$ is an upper bound on the absolute value of the second derivative of $\phi_{h}$ and $h$ is the diameter of the cell $K_{0}$. Then, if $\sum_{i=1}^{3} \Delta_{i}=0$, we simply set

$$
\phi_{h}(\boldsymbol{x})=\bar{\phi}_{K_{0}}+\sum_{i=1}^{3} \Delta_{i} \psi_{i}(\boldsymbol{x})
$$

If $\sum_{i=1}^{3} \Delta_{i} \neq 0$ we compute

$$
\sigma^{+}=\sum_{i=1}^{3} 0 \vee \Delta_{i}, \quad \sigma^{-}=\sum_{i=1}^{3} 0 \vee-\Delta_{i}
$$

and set $\theta^{+}=\min \left\{1, \sigma^{-} / \sigma^{+}\right\}$and $\theta^{-}:=\min \left\{1, \sigma^{+} / \sigma^{-}\right\}$. Then we define

$$
\phi_{h}(\boldsymbol{x})=\bar{\phi}_{K_{0}}+\sum_{i=1}^{3} \hat{\Delta}_{i} \psi_{i}(\boldsymbol{x}), \quad \text { where } \hat{\Delta}_{i}=\theta^{+}\left(0 \vee \Delta_{i}\right)-\theta^{-}\left(0 \vee-\Delta_{i}\right)
$$

The projection limiter is applied at each stage of the SSP Runge-Kutta time discretization. 


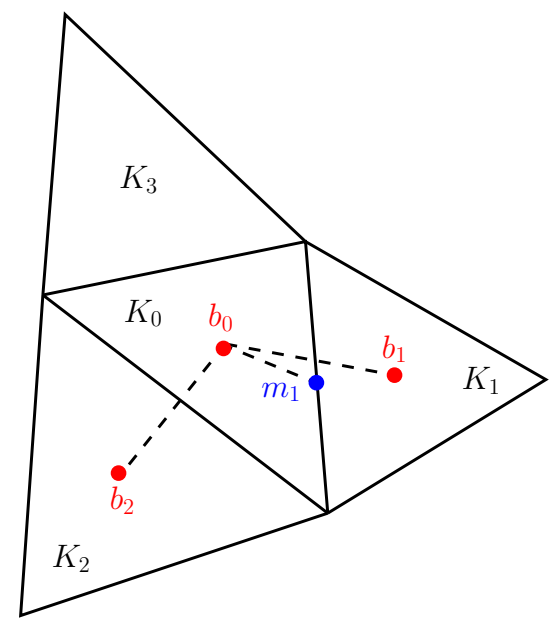

Figure 2: Sketch of the mesh entities employed in the limiting procedure.

\section{Stability analysis for the saturation equation}

Here we also assume that $\boldsymbol{u}_{h}$ is known (it has been computed in a previous iteration). If we choose $s_{h}(\boldsymbol{x}) \equiv 1$ in $S_{h}^{K}$, then 3.28 takes the form

$$
\frac{\mathrm{d}}{\mathrm{d} t} \int_{K} \phi_{h}(\boldsymbol{x}, t) \mathrm{d} \boldsymbol{x}+\sum_{\sigma \in \partial K} \int_{\sigma} \mathbf{F}\left(\phi_{h}(\boldsymbol{x}, t), \boldsymbol{u}_{h}, \boldsymbol{x}\right) \cdot \boldsymbol{n}_{K, \sigma} \mathrm{d} s=0 .
$$

We denote

$$
\bar{\phi}_{K}(t):=\frac{1}{|K|} \int_{K} \phi_{h}(\boldsymbol{x}, t) \mathrm{d} \boldsymbol{x}, \quad \bar{\phi}_{K}^{n}:=\bar{\phi}_{K}\left(t^{n}\right) .
$$

After adopting a forward Euler time stepping we end up with the equation

$$
\bar{\phi}_{K}^{n+1}=\bar{\phi}_{K}^{n}-\frac{\Delta t}{|K|} \sum_{i=1}^{3} \int_{\sigma_{i}} \mathbf{F}\left(\phi_{K}, \boldsymbol{u}_{h}, \boldsymbol{x}\right) \cdot \boldsymbol{n}_{K, \sigma_{i}} \mathrm{~d} s,
$$

where we have enumerated the edges of the triangle $K$ by $\sigma_{i}, i=1,2,3$, and $\boldsymbol{n}_{K, \sigma_{i}}$ are the corresponding normal vectors. Here the solution $\bar{\phi}_{K}^{n}$ is the average of the DG polynomial computed at time $t^{n}$, say

$$
\bar{\phi}_{K}^{n}=\frac{1}{|K|} \int_{K} p_{K}(\boldsymbol{x}) \mathrm{d} \boldsymbol{x},
$$

where $p_{K}(\boldsymbol{x})$ is the corresponding DG polynomial of degree $k$ in each of its variable. We note that if the forward Euler time discretization (4.1) satisfies the maximum principle for a time step restriction $\Delta t \leq \Delta t_{0}$, then the SSP Runge-Kutta time stepping preserves this principle for the aforementioned time step restriction (with a unitary CFL coefficient, see [27, 38]).

It is now left to verify that the DG-approximated saturation profile satisfies a maximum principle in the average value $\bar{\phi}_{K}^{n}$ for the forward Euler time discretization (4.1). In order to prove this property we modify the method adopted in [47] to the case of a discontinuous flux function. We write the average of the computed solution $\bar{\phi}_{K}^{n}$ as

$$
\bar{\phi}_{K}^{n+1}=H\left(\phi_{1,1}^{\operatorname{int}(K)}, \phi_{1,2}^{\operatorname{int}(K)}, \ldots, \phi_{3, k+1}^{\operatorname{int}(K)}, \phi_{1,1}^{\operatorname{ext}(K)}, \phi_{1,2}^{\operatorname{ext}(K)}, \ldots, \phi_{3, k+1}^{\operatorname{ext}(K)}, \phi_{1}^{\operatorname{int}}, \ldots, \phi_{M}^{\operatorname{int}}\right),
$$

where the arguments of $H$ are the DG solution computed at the quadrature points. Technical details are postponed to the Appendix. 
Lemma 4.1. The function $H$ in 4.2 is increasing in each of its variable provided the following CFL condition is satisfied

$$
\frac{\Delta t}{|K|} \sum_{i=1}^{3}\left|\sigma_{i}\right| \leq \frac{2}{3} \hat{\omega}_{1},
$$

where $\hat{\omega}_{1}$ is the first weight of the Gauss-Lobatto quadrature rule on $\left[-\frac{1}{2}, \frac{1}{2}\right]$.

Proof. See the Appendix.

Theorem 4.1. Assume that the discrete velocity is continuous across element boundaries. Then, the DG solution $\bar{\phi}_{K}^{n+1}$ computed from the saturation equation of (1.1) by using the DFLU numerical flux (3.34) satisfies the following maximum principle:

$$
0 \leq \bar{\phi}_{K}^{n+1} \leq 1
$$

provided the reconstructed $D G$ polynomial is bounded $0 \leq p_{K}(\boldsymbol{x}) \leq 1$ and that the CFL condition (4.3) is satisfied.

Proof. Lemma 4.1 states that the function $H$ is increasing in each of its variable under the CFL condition 4.3. Therefore if the reconstructed DG polynomial $p_{K}(\boldsymbol{x})$ takes values in $[0,1]$ we get

$$
H(0, \ldots, 0) \leq \bar{\phi}_{K}^{n+1} \leq H(1, \ldots, 1) .
$$

Hence, to prove (4.4) it suffices to show that $H(0, \ldots, 0)=0$ and $H(1, \ldots, 1)=1$.

Let us choose the value of the variables $\phi_{i, \beta}^{\operatorname{int}(K)}, \phi_{i, \beta}^{\operatorname{ext}(K)}$ and $\phi_{\nu}^{\text {int }}$ equal to 1 . Then we have (using the conservativity of the numerical flux)

$$
\begin{aligned}
& H_{\beta}^{1}=1-\frac{3 \Delta t}{2 \hat{\omega}_{1}|K|}\left[J\left(1,1, \boldsymbol{u}_{\beta}^{1}, \boldsymbol{x}_{\beta}^{1}, \boldsymbol{n}_{K, \sigma_{1}}\right)\left|\sigma_{1}\right|+J\left(1,1, \boldsymbol{u}_{\beta}^{1}, \boldsymbol{x}_{\beta}^{1},-\boldsymbol{n}_{K, \sigma_{1}}\right)\left|\sigma_{1}\right|\right]=1, \\
& H_{\beta}^{3}=1-\frac{3 \Delta t}{2 \hat{\omega}_{1}|K|}\left[J\left(1,1, \boldsymbol{u}_{\beta}^{3}, \boldsymbol{x}_{\beta}^{3}, \boldsymbol{n}_{K, \sigma_{3}}\right)\left|\sigma_{3}\right|+J\left(1,1, \boldsymbol{u}_{\beta}^{3}, \boldsymbol{x}_{\beta}^{3},-\boldsymbol{n}_{K, \sigma_{3}}\right)\left|\sigma_{3}\right|\right]=1, \\
& H_{\beta}^{2}=1-\frac{3 \Delta t}{2 \hat{\omega}_{1}|K|}\left[J\left(1,1, \boldsymbol{u}_{\beta}^{1}, \boldsymbol{x}_{\beta}^{1}, \boldsymbol{n}_{K, \sigma_{1}}\right)\left|\sigma_{1}\right|+J\left(1,1, \boldsymbol{u}_{\beta}^{2}, \boldsymbol{x}_{\beta}^{2}, \boldsymbol{n}_{K, \sigma_{2}}\right)\left|\sigma_{2}+J\left(1,1, \boldsymbol{u}_{\beta}^{3}, \boldsymbol{x}_{\beta}^{3}, \boldsymbol{n}_{K, \sigma_{3}}\right)\right| \sigma_{3} \mid\right] .
\end{aligned}
$$

Therefore

$$
\begin{aligned}
H(1, \ldots, 1)= & \sum_{\nu=1}^{M} \tilde{\omega}_{\nu}+\sum_{\beta=1}^{k+1} \frac{2}{3} \omega_{\beta} \hat{\omega}_{1}\left(3-\frac{3 \Delta t}{2 \hat{\omega}_{1}|K|}\left[J\left(1,1, \boldsymbol{u}_{\beta}^{1}, \boldsymbol{x}_{\beta}^{1}, \boldsymbol{n}_{K, \sigma_{1}}\right)\left|\sigma_{1}\right|\right.\right. \\
& \left.\left.+J\left(1,1, \boldsymbol{u}_{\beta}^{2}, \boldsymbol{x}_{\beta}^{2},-\boldsymbol{n}_{K, \sigma_{2}}\right)\left|\sigma_{2}+J\left(1,1, \boldsymbol{u}_{\beta}^{3}, \boldsymbol{x}_{\beta}^{3}, \boldsymbol{n}_{K, \sigma_{3}}\right)\right| \sigma_{3} \mid\right]\right) \\
= & \sum_{\nu=1}^{M} \tilde{\omega}_{\nu}+\sum_{\beta=1}^{k+1} 2 \omega_{\beta} \hat{\omega}_{1}-\frac{\Delta t}{|K|} \sum_{\beta=1}^{k+1}\left[J\left(1,1, \boldsymbol{u}_{\beta}^{1}, \boldsymbol{x}_{\beta}^{1}, \boldsymbol{n}_{K, \sigma_{1}}\right)\left|\sigma_{1}\right|\right. \\
& \left.\quad+J\left(1,1, \boldsymbol{u}_{\beta}^{2}, \boldsymbol{x}_{\beta}^{2}, \boldsymbol{n}_{K, \sigma_{2}}\right)\left|\sigma_{2}+J\left(1,1, \boldsymbol{u}_{\beta}^{3}, \boldsymbol{x}_{\beta}^{3}, \boldsymbol{n}_{K, \sigma_{3}}\right)\right| \sigma_{3} \mid\right] \omega_{\beta} \\
= & 1-\frac{\Delta t}{|K|} \sum_{i=1}^{3} \sum_{\beta=1}^{k+1} J\left(1,1, \boldsymbol{u}_{\beta}^{i}, \boldsymbol{x}_{\beta}^{i}, \boldsymbol{n}_{K, \sigma_{i}}\right)\left|\sigma_{i}\right| \omega_{\beta},
\end{aligned}
$$

where we have used the equality $\sum_{\nu=1}^{M} \tilde{\omega}_{\nu}+\sum_{\beta=1}^{k+1} 2 \omega_{\beta} \hat{\omega}_{1}=1$, which is obtained from A.5 (see Appendix) if we choose the polynomial $p_{K} \equiv 1$. 
The second term in the right-hand side of 4.5 indicates the quadrature rule for the flux $\mathbf{F}$, so that

$$
\begin{aligned}
\sum_{i=1}^{3} \sum_{\beta=1}^{k+1} J\left(1,1, \boldsymbol{u}_{\beta}^{i}, \boldsymbol{x}_{\beta}^{i}, \boldsymbol{n}_{K, \sigma_{i}}\right)\left|\sigma_{i}\right| \omega_{\beta} & =\sum_{i=1}^{3} \sum_{\beta=1}^{k+1} \mathbf{F}\left(1, \boldsymbol{u}_{\beta}^{i}, \boldsymbol{x}_{\beta}^{i}\right) \cdot \boldsymbol{n}_{K, \sigma_{i}}\left|\sigma_{i}\right| \omega_{\beta}=\sum_{i=1}^{3} \int_{\sigma_{i}} \mathbf{F}\left(1, \boldsymbol{u}_{h}, \boldsymbol{x}\right) \cdot \boldsymbol{n}_{K, \sigma_{i}} \mathrm{~d} s \\
& =\int_{\partial K} \mathbf{F}\left(1, \boldsymbol{u}_{h}, \boldsymbol{x}\right) \cdot \boldsymbol{n} \mathrm{d} s=\int_{\partial K}\left(\boldsymbol{u}_{h}+b(1) K(\boldsymbol{x}) \boldsymbol{g}\right) \cdot \boldsymbol{n} \mathrm{d} s \\
& =\int_{K} \nabla \cdot \boldsymbol{u}_{h} \mathrm{~d} \boldsymbol{x}=0,
\end{aligned}
$$

where we have used the relation $b(1) \equiv 0$ and have applied integration by parts to the DG solution $\boldsymbol{u}_{h}$ in the triangle $K$. Thus from 4.5 we get $H(1, \ldots, 1)=1$. A similar argument shows that $H(0, \ldots, 0)=0$, which completes the proof.

Remark 4.1. Notice that the discrete velocity generated with the DFVE method is not necessarily continuous across element boundaries. Then, an intermediate projection step (from the discrete trial velocity space to primal edge-wise continuous functions) would be required to rigorously derive the result stated in Theorem 4.1. However, the computational results in Section 5 indicate that such a restriction may be only technical.

Finally the maximum principle in the mean approximated solution of the saturation equation follows if the DG polynomial satisfies the condition $p_{K}(\boldsymbol{x}) \in[0,1]$ for all $\boldsymbol{x} \in \hat{S}_{K}\left(\hat{S}_{K}\right.$ is defined in the Appendix). To enforce this condition it is necessary to apply a simple linear scaling limiter as in [47. So with the assumption that $\bar{\phi}_{K}^{n} \in[0,1]$ for all $K$, we use the following modified $\tilde{p}_{K}(\boldsymbol{x})$ instead of $p_{K}(\boldsymbol{x})$ :

$$
\tilde{p}_{K}(\boldsymbol{x})=\theta\left(p_{K}(\boldsymbol{x})-\bar{\phi}_{K}^{n}\right)+\bar{\phi}_{K}^{n}, \quad \theta=\min \left\{\left|\frac{1-\bar{\phi}_{K}^{n}}{M_{K}-\bar{\phi}_{K}^{n}}\right|,\left|\frac{\bar{\phi}_{K}^{n}}{m_{K}-\bar{\phi}_{K}^{n}}\right|, 1\right\},
$$

where

$$
M_{K}:=\max _{\boldsymbol{x} \in \hat{S}_{K}} p_{K}(\boldsymbol{x}), \quad m_{K}:=\min _{\boldsymbol{x} \in \hat{S}_{K}} p_{K}(\boldsymbol{x})
$$

It is easy to verify that the reconstructed polynomial $\tilde{p}_{K}(\boldsymbol{x})$ assumes values in $[0,1]$ and satisfies the conservativity property $\frac{1}{|K|} \int_{K} \tilde{p}_{K}(\boldsymbol{x}) \mathrm{d} \boldsymbol{x}=\bar{\phi}_{K}^{n}$. In addition, the above linear scaling limiter preserves the accuracy of the scheme, which can be verified along the lines of [48].

\section{Numerical results}

At each time step, a fixed-point method has been applied to couple of the Brinkman problem with the nonlinear transport equation. A fixed tolerance of $10^{-6}$ is imposed on the energy residual of each sub-problem. In addition, the linear systems involved have been solved by a preconditioned conjugate gradient method.

\subsection{Example 1: accuracy of the DFVE formulation for smooth solutions and continuous fluxes}

We start by assessing the convergence of the proposed DFVE scheme applied to a non-homogeneous version of problem (1.1) admitting the following exact solutions:

$$
\boldsymbol{u}=\sin (t)\left(\begin{array}{c}
\sin \left(2 \pi x_{1}\right) \cos \left(2 \pi x_{2}\right) \\
-\cos \left(2 \pi x_{1}\right) \sin \left(2 \pi x_{2}\right)
\end{array}\right), \quad p=\cos (t)\left(x_{1}^{2}+x_{2}^{2}-\frac{2}{3}\right), \quad \phi=\sin (t) \sin \left(\pi x_{1}\right) \sin \left(\pi x_{2}\right) .
$$

The momentum equation now possesses a forcing term $\boldsymbol{r}_{\boldsymbol{u}}$ and the saturation transport equation has a nonzero right-hand side $r_{\phi}$ computed using these manufactured smooth solutions. Initial and Dirichlet 


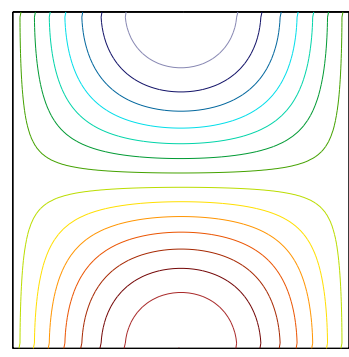

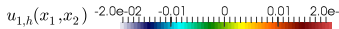

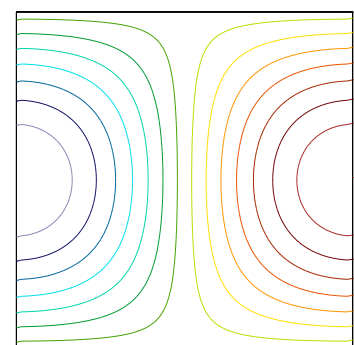

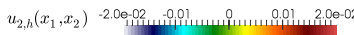

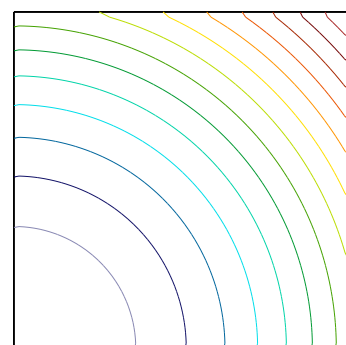

$p_{h}\left(x_{1}, x_{2}\right) \quad-6.70-01, \quad 01$

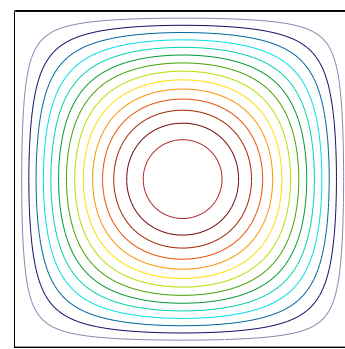

$\phi_{h}\left(x_{1}, x_{2}\right)^{3.99-060.004}$ 0.008 $0.0120 .0162 .00-0$

Figure 3: Example 1: approximate solutions for the convergence test of the DFVE formulation of 1.1 with continuous flux.

boundary data are also imposed according to the exact solutions. In this test it is assumed that $\mathbf{K}=K \mathbf{I}$ is constant on $\Omega$ and the following model and stabilization parameters are adopted: $K=10, \alpha_{d}=1000$. Moreover, the saturation-dependent coefficients are $\mu(\phi)=\left(1-\frac{1}{2} \phi\right)^{-2}, f(\phi)=\phi, b(\phi)=\frac{1}{K} \phi^{3}\left(1-\frac{1}{2} \phi\right)^{2}$.

To study the spatial accuracy we fix a time step $\Delta t=0.0005$, evolve the system until $T=1$, and compute errors between the approximate and exact solutions in the natural norms. The obtained error history, including rates of convergence is displayed in the first part of Table 1 . To this end, we would like to mention that even though we could not establish any theoretical error estimates for the saturation $\phi$ (see Remark 3.2), we observe from the computational results a linear rate of convergence (for the spatial discretization) for $\left\|\phi-\phi_{h}\right\|_{h}$, measured in a scalar version of the norm $\|\cdot\|_{h}$ defined in (3.11). We also test the accuracy of the time advancing scheme by computing discrete $\ell^{\infty}$ errors on uniform nested discretizations of the time interval $[0, T]$ into $N-1$ segments, associated to a fixed fine mesh with size $h=0.006743$. Moreover, we report a rough average of nine fixed-point iterations to reach the desired tolerance. The approximate solutions at the final time $T=1$ are depicted in Figure 3 .

For all remaining examples, the parameter $\hat{M}$ (needed in the flux limiting procedure detailed in Section 3.6 is taken as the global bound for the discrete seminorm of the initial saturation. This value (which does not correspond necessarily with its theoretical value, rarely available analytically) yields satisfactory results in terms of accuracy and performance.

\subsection{Example 2: viscous flow on a porous square with discontinuous absolute permeability}

In this test the domain coincides with the one in the previous example but we consider a heterogeneous medium composed by two different materials separated by a vertical line $x_{1}=a$, and characterized by isotropic absolute permeabilities

$$
\mathbf{K}(\boldsymbol{x})= \begin{cases}\mathbf{I} & \text { if } x_{1}<a, \\ 3 \mathbf{I} & \text { if } x_{1}>a .\end{cases}
$$

In addition, the following saturation-dependent and constant coefficients are adopted:

$$
\begin{aligned}
K_{\mathrm{w}}(\phi) & =\phi, \quad K_{\mathrm{n}}(\phi)=1-\phi, \quad f(\phi)=\phi, \quad b(\phi)=\Delta \rho \phi(1-\phi), \quad \mu(\phi)=\mu_{0}(1-\phi / 2)^{-\eta}, \\
\mu_{\mathrm{w}} & =\mu_{\mathrm{n}}=\phi_{-}=1, \quad \boldsymbol{g}=(0,-1)^{\mathrm{T}}, \quad \eta=2.5, \quad a=0.5, \quad \phi_{+}=0, \quad \Delta \rho=0.1, \quad \mu_{0}=0.01 .
\end{aligned}
$$

The initial saturation distribution corresponds to

$$
\phi_{0}(\boldsymbol{x})= \begin{cases}\phi_{-} & \text {if } x_{1}<a, \\ \phi_{+} & \text {if } x_{1}>a .\end{cases}
$$

On $\partial \Omega$ we assume zero-flux boundary conditions for the saturation, and slip (zero normal) velocity (see Figure 4, left). The pressure is penalized so that $p \in L_{0}^{2}(\Omega)$. At constant velocity $\boldsymbol{u}=(1,1)^{\mathrm{T}}$, the left 


\begin{tabular}{|c|c|c|c|c|c|c|c|}
\hline$h$ & $\left\|\phi(T)-\phi_{h}^{N}\right\|_{h}$ & rate & $\left\|\boldsymbol{u}(T)-\boldsymbol{u}_{h}^{N}\right\|_{h}$ & rate & $\left\|p(T)-p_{h}^{N}\right\|_{0, \Omega}$ & rate & iter \\
\hline 0.35355 & 0.01619 & - & 0.09594 & - & 0.43608 & - & 11 \\
\hline 0.28284 & 0.01265 & 1.10516 & 0.07909 & 0.86508 & 0.37494 & 0.87699 & 9 \\
\hline 0.20203 & 0.00879 & 1.08285 & 0.05829 & 0.90720 & 0.28577 & 0.90714 & 8 \\
\hline 0.12856 & 0.00544 & 1.05862 & 0.03867 & 0.90753 & 0.19163 & 0.98406 & 9 \\
\hline 0.07395 & 0.00308 & 0.99246 & 0.02153 & 0.95421 & 0.11753 & 0.99167 & 8 \\
\hline 0.03921 & 0.00189 & 0.99540 & 0.01344 & 0.99742 & 0.07304 & 0.99308 & 10 \\
\hline$\Delta t$ & $\phi\left(t^{n}\right)-\phi_{h}^{n} \|_{h}$ & rate & $\boldsymbol{u}\left(t^{n}\right)-\boldsymbol{u}_{h}^{n} \|_{h}$ & rate & $\left(t^{n}\right)-p_{h}^{n} \|_{0, \Omega}$ & rate & avg. iter \\
\hline 0.50000 & 0.42634 & - & 0.77506 & - & 1.76558 & - & 8 \\
\hline 0.12500 & 0.11711 & 1.80678 & 0.24867 & 1.60325 & 0.57560 & 1.56932 & 10 \\
\hline 0.03125 & 0.02725 & 1.90584 & 0.05687 & 1.78043 & 0.20112 & 1.57750 & 8 \\
\hline 0.00625 & 0.00597 & 1.98743 & 0.01508 & 1.90291 & 0.05319 & 1.96023 & 8 \\
\hline 0.00156 & 0.00153 & 2.01031 & 0.03674 & 1.97425 & 0.01319 & 1.95383 & 7 \\
\hline 0.00078 & 0.00049 & 1.99134 & 0.01091 & 1.93908 & 0.00356 & 1.96402 & 9 \\
\hline
\end{tabular}

Table 1: Example 1: convergence results for the DFVE approximation of 1.1, computed on a sequence of uniformly refined partitions of $\Omega=(0,1)^{2}$ (top rows), and $\ell^{\infty}$ errors, observed convergence rates, and Picard iteration counts (total for the space-refined tests, and average for the time-refined ones) for successively refined time intervals using a RK2 time stepping. First and second order convergences are evidenced in each case.

and right branches of the flux components (denoted by the respective superscripts "_" and "+") behave as depicted in the right panel of Figure 4. Notice that the horizontal component of the flux function is continuous for any saturation value. For this example the numerical flux is chosen as in (3.34) and the domain is discretized by 10205 points forming a primal mesh of 20408 structured triangles. A fixed time step of $\Delta t=0.005$ is employed.

Initially, the interface between phases $\mathrm{w}$ and $\mathrm{n}$ is aligned with the discontinuity of the medium. Thereafter, the sharp interface ends up aligned perpendicular to the permeability discontinuity and parallel to gravity. Both pressure and velocity profiles seem to be influenced by the moving interface rather than by the domain discontinuity (see Figure 5).

\subsection{Example 3: flow on a quarter-five spot domain with discontinuous relative and absolute permeability}

We now focus on a more involved discontinuity where also the relative permeability of one phase changes from one material type to the other. The computational domain now corresponds to the upper right quarter of the well-known five spot geometry, that is, $\Omega=(0,1)^{2} \backslash\left(B_{0.075}(0,0) \cup B_{0.075}(1,1)\right)$, where $B_{r}\left(\boldsymbol{x}^{c}\right)$ denotes the ball of radius $r$ centered at a given point $\boldsymbol{x}^{c}=\left(x_{1}^{c}, x_{2}^{c}\right)$. The domain is initially at rest and with constant saturation $\phi=\phi_{0}$ on the whole domain. Boundary conditions will be configured as in (2.1): an inlet section (or injector site) $\Gamma_{\text {in }}$ corresponds to the bottom left corner (see the domain sketch in Figure 6), where we inject material with a low constant saturation $\phi=\phi_{\text {in }}$ at a normal inflow velocity of $\boldsymbol{u} \cdot \boldsymbol{n}=u_{\mathrm{in}}$. At the outlet $\Gamma_{\text {out }}$ (the so-called producer well, located at the top right corner of the domain) we set a constant pressure value $p=p_{\text {out }}$, whereas on the walls $\Gamma_{\text {wall }}=\partial \Omega \backslash\left(\Gamma_{\text {in }} \cup \Gamma_{\text {out }}\right)$ (the remainder of the boundary), slip velocities $(\boldsymbol{u} \cdot \boldsymbol{n}=0)$ are allowed and the saturation satisfies a zero-flux boundary condition.

We choose $a, \mu(\phi), \mu_{\mathrm{w}}, \mu_{\mathrm{n}}$ as in the previous example. On the other hand, we impose

$$
\mathbf{K}(\boldsymbol{x})=\left\{\begin{array}{ll}
2 \mathbf{I} & \text { if } x_{1}<a, \\
\mathbf{I} & \text { if } x_{1}>a,
\end{array} \quad K_{\mathrm{w}}^{-}(\phi)=\left\{\begin{array}{ll}
1.75 \phi & \text { if } \phi \leq 0.25, \\
0.25 \phi+0.375 & \text { if } \phi>0.25,
\end{array} \quad K_{\mathrm{w}}^{+}(\phi)=\phi,\right.\right.
$$




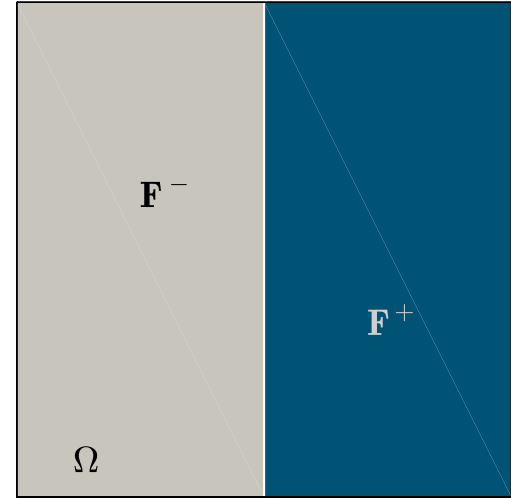

$\partial \Omega: u \cdot n=0, b(\phi) \mathbf{K} g \cdot n=0$

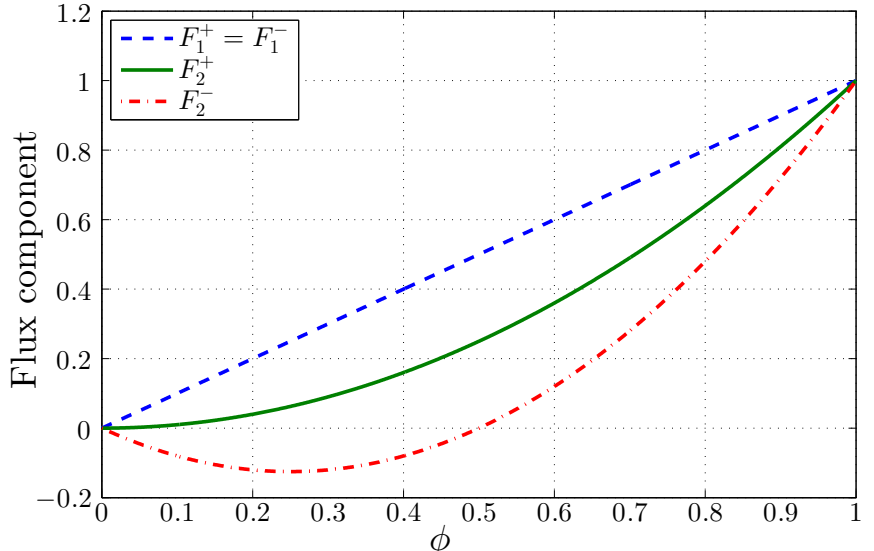

Figure 4: Example 2: domain, material discontinuity, and boundary condition (left panel), and left and right branches of the discontinuous flux components for a constant velocity $\boldsymbol{u}=(1,1)^{\mathrm{T}}$ (right).

$$
\begin{aligned}
K_{\mathrm{n}}(\phi) & =1-\phi^{2}, \quad f(\phi)=\frac{K_{\mathrm{w}}(\phi)}{K_{\mathrm{w}}(\phi)+K_{\mathrm{n}}(\phi)}, \quad \mathbf{F}(\phi, \boldsymbol{u})=f(\phi) \boldsymbol{u}, \\
\phi_{\text {in }} & =0.02, \quad \phi_{0}=0.75, \quad u_{\text {in }}=-0.1, \quad p_{\text {out }}=2, \quad \rho_{\mathrm{w}}=20, \quad \rho_{\mathrm{n}}=10 .
\end{aligned}
$$

For a constant value of the velocity field we have plotted the branches of the horizontal and vertical flux components in Figure 6 .

Snapshots of the approximate saturation, velocity and pressure are shown in Figure 7 We observe that the low saturation fluid is flowing from the inlet towards the outlet following a symmetric pattern. This example, as well as Example 4, illustrate that the method is suitable for long-time simulations of realistic scenarios. In particular saturation fronts are sharply resolved, with saturations assuming physically relevant values between zero and one.

\subsection{Example 4: viscous flow on a porous square with irregular absolute permeability}

We keep the same computational domain as in Example 2 and we focus on the case of a nonhomogeneous absolute permeability $\mathbf{K}=K(\boldsymbol{x}) \mathbf{I}$, with

$$
K(\boldsymbol{x})= \begin{cases}5.0 \times 10^{-6} & \text { if } \boldsymbol{x} \in \mathrm{B}_{0.0015}\left(x_{1}^{i}, x_{2}^{i}\right) \text { for } i \in\{1, \ldots, N\}, \\ 1.5 \times 10^{-5} & \text { otherwise. }\end{cases}
$$

Here $\boldsymbol{x}_{i}$ are $N=40$ randomly chosen points on $\Omega$ (see e.g. [35]). Moreover, the viscosities and relative permeabilities are

$$
\mu_{\mathrm{w}}=0.2, \quad \mu_{\mathrm{n}}=1, \quad K_{\mathrm{w}}(\phi)=\phi^{2}, \quad K_{\mathrm{n}}(\phi)=(1-\phi)^{2},
$$

whereas the remaining parameters are kept as in Example 2. The domain is initially at homogeneous saturation of 0.02 . On the left wall we inject fluid of saturation 0.8 at an inflow velocity of 0.001 . An horizontal gravity field is considered $\boldsymbol{g}=(0.0098,0)^{\mathrm{T}}$. On the outlet (right wall) we set a zero pressure. Figure 8 shows the approximate velocity and pressure fields at the final adimensional time $T=500$, along with snapshots of the approximate saturation. We observe a smooth front forms going from left to right, where saturation profiles are slightly affected by the permeability heterogeneities. 

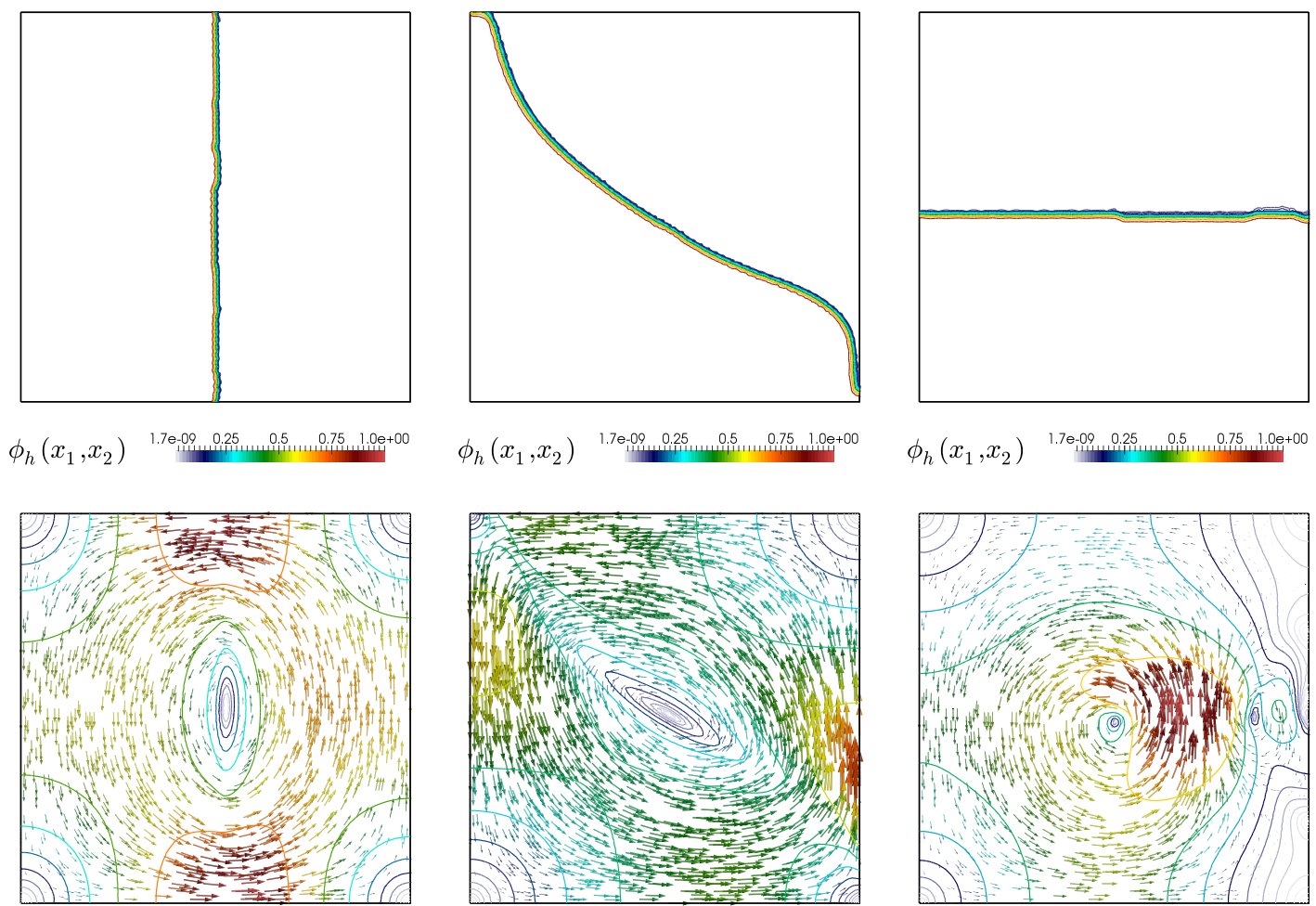

$u_{h}\left(x_{1}, x_{2}\right)^{1.8 \mathrm{e}-02} 0.20 .30 .45 .4 \mathrm{e}-0$
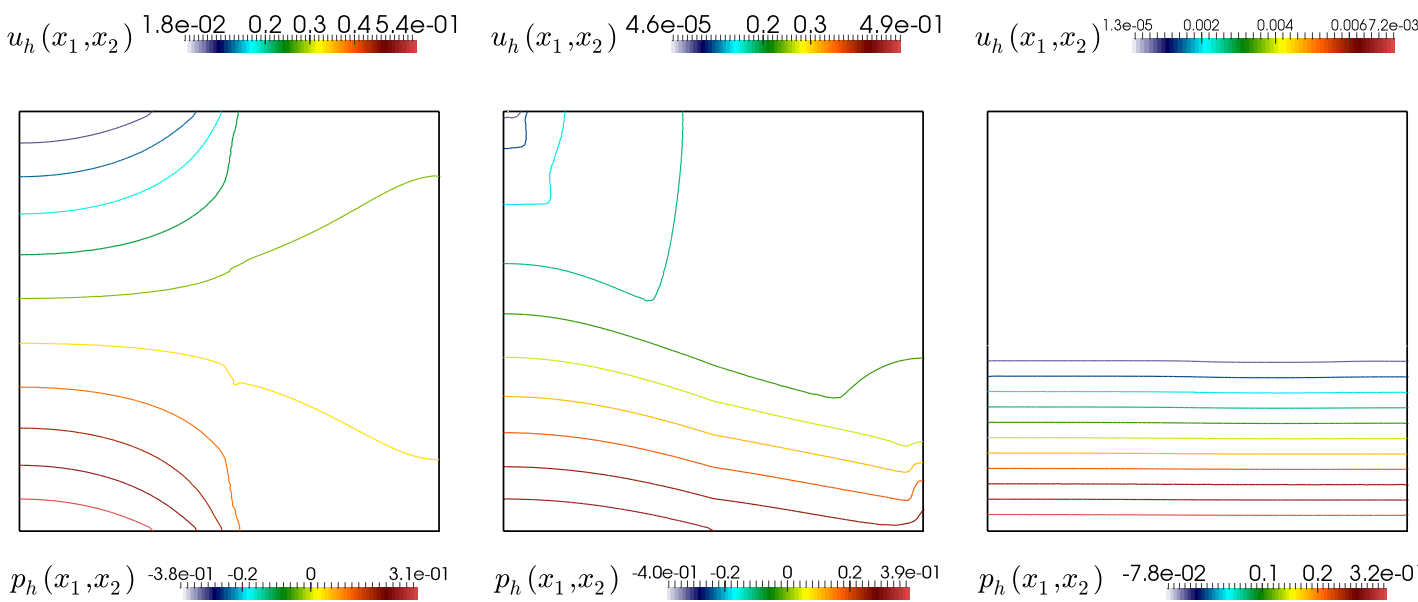

$p_{h}\left(x_{1}, x_{2}\right)^{-4.0 \mathrm{e}-01}-0.2 \quad 0 \quad 0.2 \quad 3.9 \mathrm{e}-01$

$p_{h}\left(x_{1}, x_{2}\right) \quad-7.8 \mathrm{e}-02 \quad 0.1 \quad 0.2 \quad 3.2 \mathrm{e}-01$

Figure 5: Example 2: approximate saturation (top row), velocity vectors and contours of velocity magnitude (middle), and pressure contours (bottom panels), computed with the DFVE-RKDG scheme on a structured mesh of 20408 primal triangular elements, shown at early (left), middle (center), and advanced (right column) time instants.

\subsection{Example 5: viscous flow on a porous square with high-contrast, irregular absolute permeability}

For our final test case we consider a wider domain $\Omega=(0,2) \times(0,1)$ with a higher-contrast permeability field given by $\mathbf{K}=K(\boldsymbol{x}) \mathbf{I}$, with $K(\boldsymbol{x})=10^{-7}+10^{-2}\left|\sum_{i=0}^{N} \Phi_{i}(\boldsymbol{x})\right|$, where $N=90$ and $\Phi_{i}(\boldsymbol{x})=\exp \left(-400\left|\boldsymbol{x}-\boldsymbol{x}^{i}\right|^{2}\right)$. Viscosities, relative permeabilities, and all remaining parameters are set as in Example 4. Zero initial velocity, pressure, and homogeneous saturation are assumed in the reservoir. On the left wall (considered as inflow boundary) we now impose a constant pressure profile $p=0.01$ along with a high saturation $\phi=0.9$, whereas at the outlet (right side) we set a constant non-homogeneous Neumann 

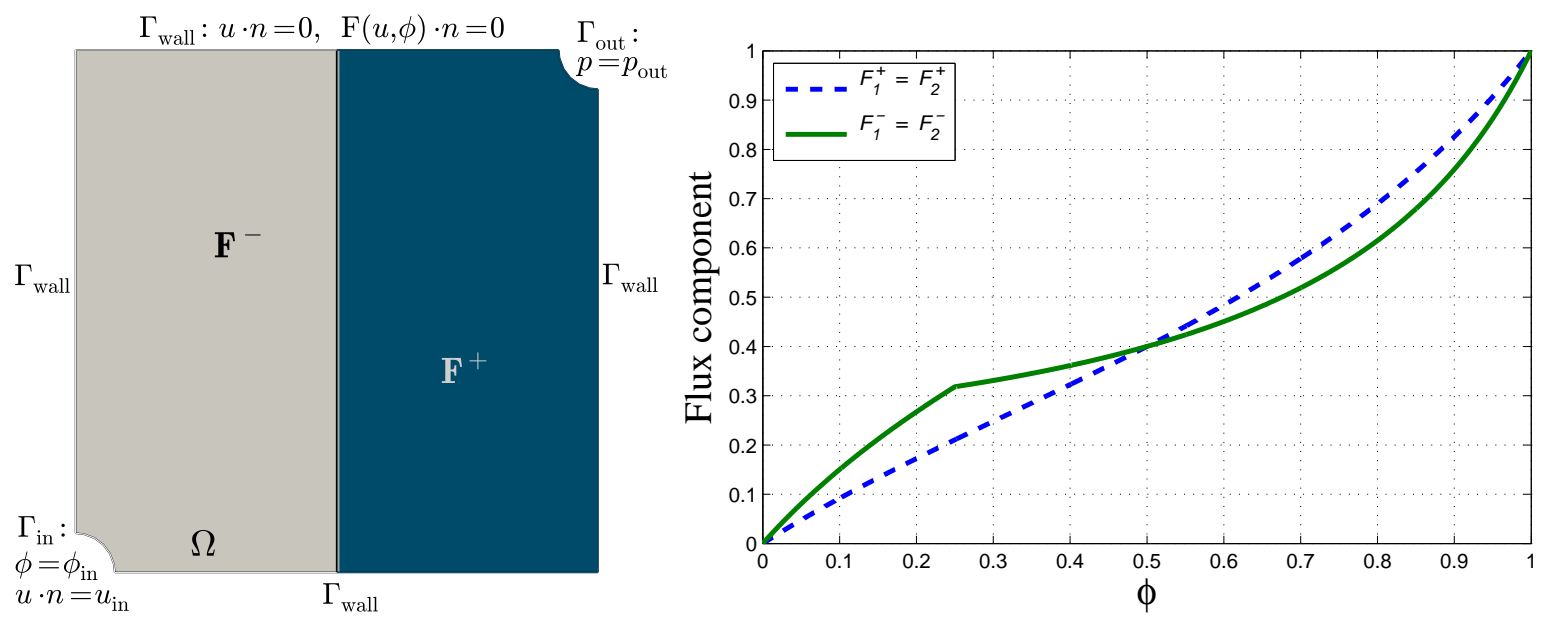

Figure 6: Example 3: domain, flux discontinuity, and boundary configurations (left panel), and left and right branches of the discontinuous flux components for a constant velocity $\boldsymbol{u}=(1,1)^{\mathrm{T}}$ (right).
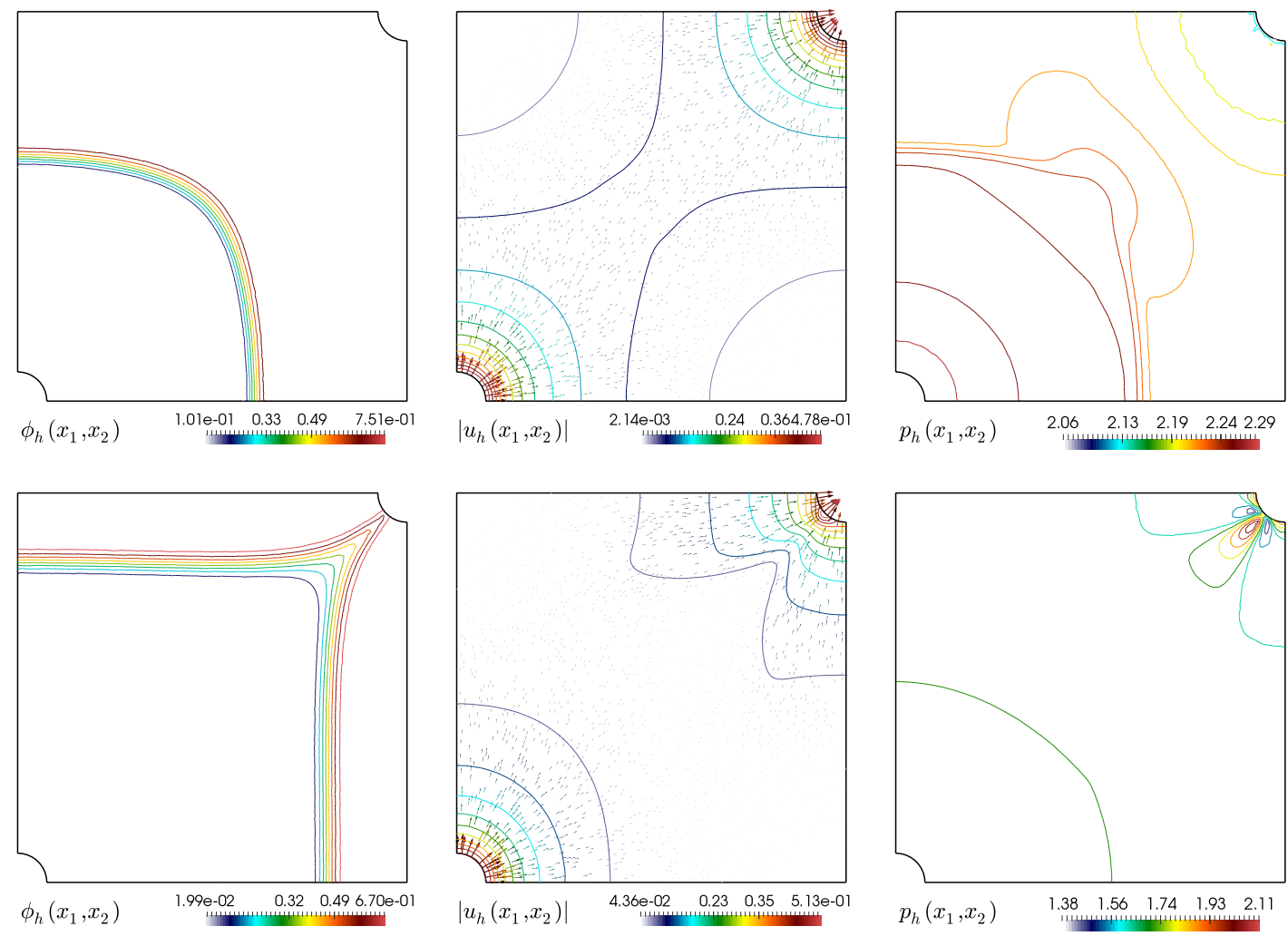

Figure 7: Example 3: approximate saturation (left), velocity vectors and contours of velocity magnitude (middle), and pressure contours (right), computed with the DFVE-RKDG scheme on an unstructured mesh of 7809 vertices and 15616 primal triangular elements, shown at middle (top), and advanced (bottom panels) time instants.

boundary condition (defined e.g. by fixing a normal outflow velocity $\boldsymbol{u} \cdot \boldsymbol{n}=0.001$ ). The top and bottom sides are considered as fixed walls. Again we consider an horizontal gravity force $\boldsymbol{g}=(0.098,0)^{\mathrm{T}}$. The 

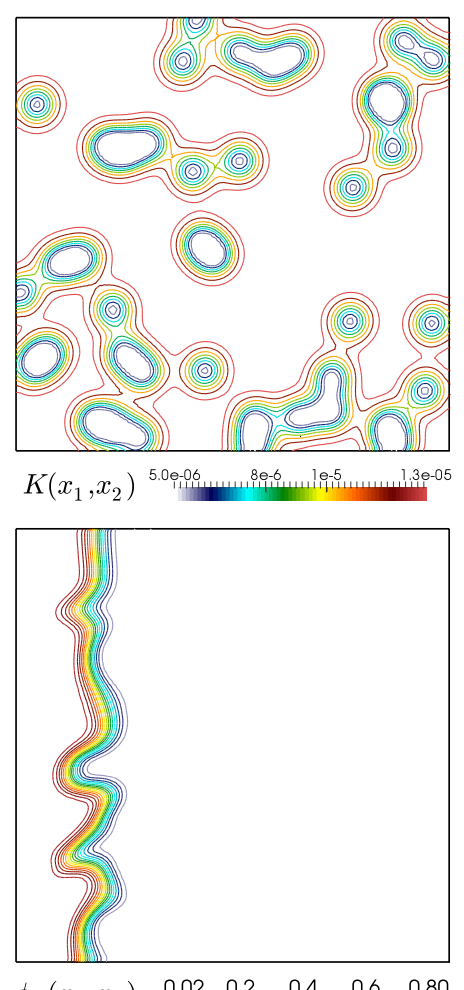

$\phi_{h}\left(x_{1}, x_{2}\right) \quad 0.02 \quad 0.2 \quad 0.4 \quad 0.6 \quad 0.80$
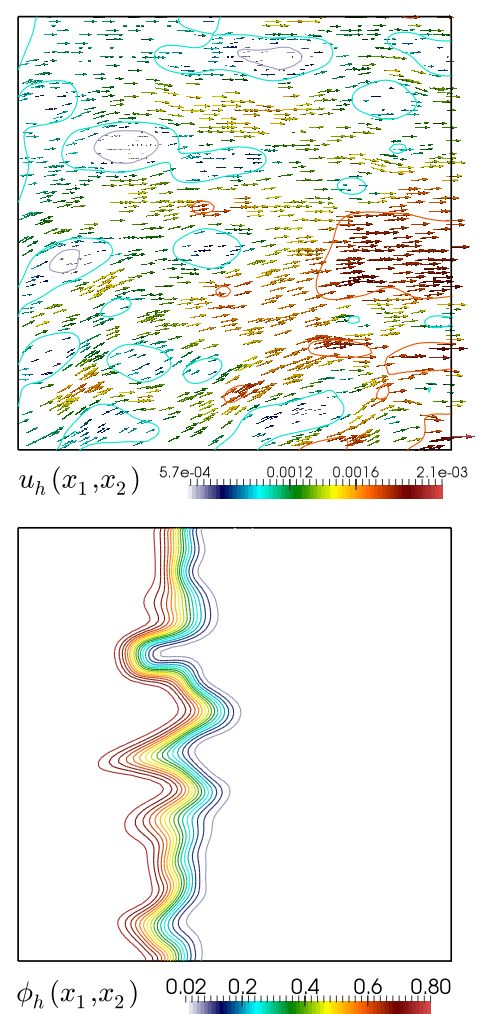
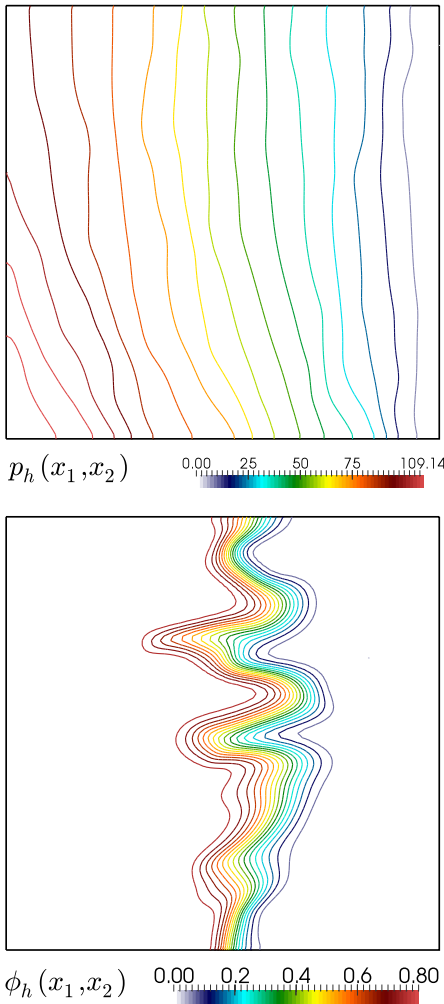

Figure 8: Example 4: Permeability field (top left), approximate velocity vectors and magnitude contour plots (top center) and pressure profiles computed at an advanced time (top right), and snapshots of the saturation at early (left), middle (center), and advanced (right column) time instants.

domain is partitioned into 32940 unstructured primal triangular elements and the timestep is $\Delta t=0.05$. Figure 9 depicts the numerical results corresponding to transients at $t=200$ and $t=600$. The pressure gradient is oriented from source (left) to sink (right) and channels of preferential velocity form due to the permeability heterogeneities and flow conditions. At earlier times, the flow patterns are more visible (due to the initial high differences between the injected saturation and the constant content in the reservoir).

\section{Conclusions}

We have presented a combined DFVE-RKDG discretization for solving two-phase flow problems involving viscous fluid within heterogeneous porous media. The problem is formulated in terms of Brinkman velocity and pressure, coupled with saturation under fractional flow, and discontinuous fluxes are used. An accurate Godunov-type flux reconstruction is employed along with a robust limiting procedure. Solutions obtained by the proposed scheme satisfy a discrete maximum principle and a few examples illustrate some properties of the method. Let us stress that the generalization of both continuous analysis and discrete formulation to the three-dimensional case is straightforward, following e.g. [12]. Also, extensions to other FVE stable formulations for flow can be incorporated without much effort (cf. [14, 31, 33]). High order saturation approximations and different numerical fluxes especially tailored for discontinuous fluxes could be also employed without affecting the coupling structure. Planned extensions to this work include the study of two-phase multi-component polymer flooding using the formulation introduced herein, the sedimentation of polydisperse suspensions, and the design of fully high-order methods. 

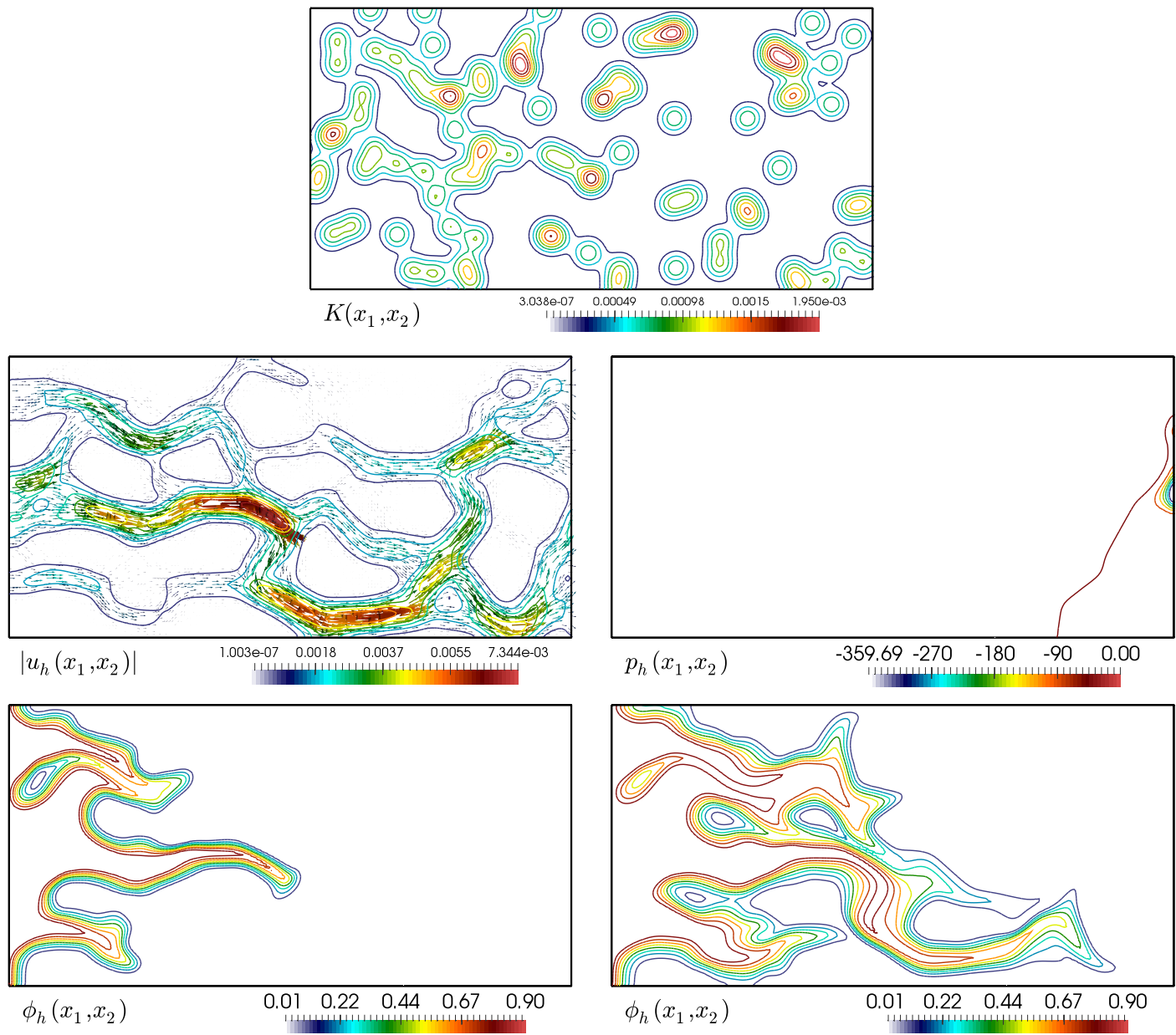

Figure 9: Example 5: Permeability distribution (top), snapshots of velocity and pressure (middle left and middle right) at $t=200$, and saturation profiles at $t=200$ and and $t=600$ (bottom row).

\section{Acknowledgments}

RB has been supported by Fondecyt project 1130154; BASAL project CMM, Universidad de Chile and Centro de Investigación en Ingeniería Matemática $\left(\mathrm{CI}^{2} \mathrm{MA}\right)$, Universidad de Concepción; Conicyt project Anillo ACT1118 (ANANUM); Red Doctoral REDOC.CTA, MINEDUC project UCO1202; CRHIAM, project CONICYT/FONDAP/15130015; and Fondef project ID15I10291. SKK gratefully acknowledges the financial support by the Fondecyt project 3150313; SK and RRB acknowledge the research support by the Department of Science and Technology, Government of India, through the National Programme on Differential Equations: Theory, Computation and Applications; and RRB acknowledges the support by the Elsevier Mathematical Sciences Sponsorship Fund.

\section{A. Appendix}

In this appendix we present the technical details for obtaining the stability results for saturation equation. The term $\mathbf{F}\left(\phi_{h}(\boldsymbol{x}, t), \boldsymbol{u}_{h}(\boldsymbol{x}), x\right) \cdot \boldsymbol{n}_{K, \sigma_{i}}$ in the integral is approximated by the numerical flux as

$$
\mathbf{F}\left(\phi_{h}(\boldsymbol{x}, t), \boldsymbol{u}_{h}(\boldsymbol{x}), x\right) \cdot \boldsymbol{n}_{K, \sigma_{i}} \approx J\left(\phi_{h}\left(\boldsymbol{x}^{\mathrm{int}(K)}, t\right), \phi_{h}\left(\boldsymbol{x}^{\operatorname{ext}(K)}, t\right), \boldsymbol{u}_{h}(\boldsymbol{x}), \boldsymbol{x}, \boldsymbol{n}_{K, \sigma_{i}}\right) .
$$




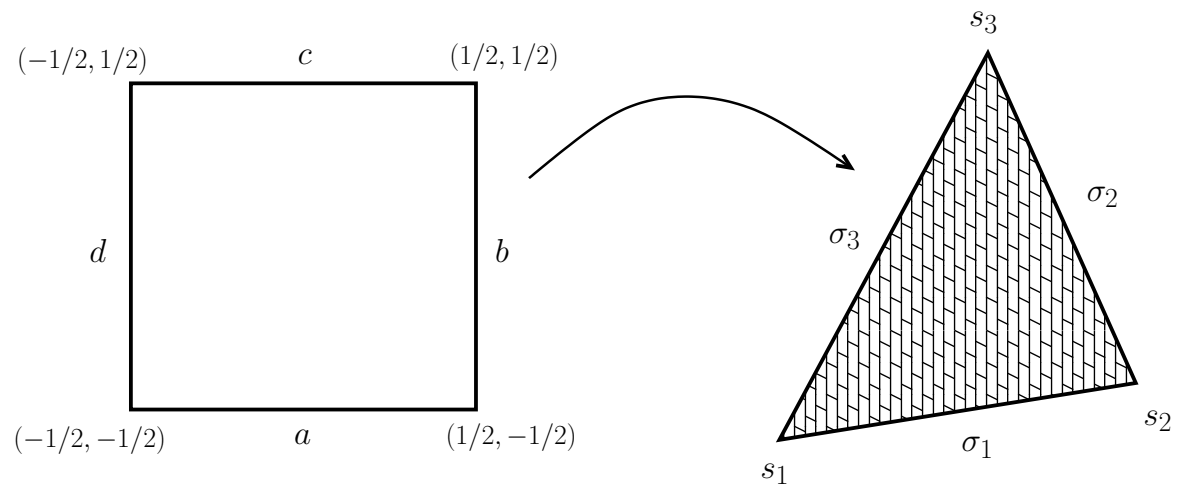

Figure 10: Representation of the projection map between the rectangular and triangular reference elements.

Inserting this into equation 4.1 yields

$$
\bar{\phi}_{K}^{n+1}=\bar{\phi}_{K}^{n}-\frac{\Delta t}{|K|} \sum_{i=1}^{3} \int_{\sigma_{i}} J\left(\phi_{h}\left(\boldsymbol{x}^{\operatorname{int}(K)}, t\right), \phi_{h}\left(\boldsymbol{x}^{\operatorname{ext}(K)}, t\right), \boldsymbol{u}_{h}(\boldsymbol{x}), \boldsymbol{x}, \boldsymbol{n}_{K, \sigma_{i}}\right) \mathrm{d} s .
$$

We need to approximate the edge integral in A.1 by a quadrature rule which is exact for polynomial of degree $(2 k+1)$ (see [18, Proposition 2.1]), for which we use the $(k+1)$-point Gauss quadrature rule. Let $\left\{\boldsymbol{x}_{\beta}^{i}, \beta=1, \ldots, k+1\right\}$ denote the set of quadrature points corresponding to the edges $\sigma_{i}, i=1,2,3$. Also we use the following notation:

$$
\phi_{i, \beta}^{\operatorname{int}(K)}=\phi_{h}\left(\boldsymbol{x}_{\beta}^{i^{\operatorname{int}(K)}}\right), \quad \phi_{i, \beta}^{\operatorname{ext}(K)}=\phi_{h}\left(\boldsymbol{x}_{\beta}^{i \operatorname{ext}(K)}\right), \quad \boldsymbol{u}_{\beta}^{i}=\boldsymbol{u}_{h}\left(\boldsymbol{x}_{\beta}^{i}\right) .
$$

Thus, the formulation A.1 reduces to

$$
\bar{\phi}_{K}^{n+1}=\bar{\phi}_{K}^{n}-\frac{\Delta t}{|K|} \sum_{i=1}^{3} \sum_{\beta=1}^{k+1} J\left(\phi_{i, \beta}^{\operatorname{int}(K)}, \phi_{i, \beta}^{\operatorname{ext}(K)}, \boldsymbol{u}_{\beta}^{i}, \boldsymbol{x}_{\beta}^{i}, \boldsymbol{n}_{K, \sigma_{i}}\right) \omega_{\beta}\left|\sigma_{i}\right|,
$$

where $\left|\sigma_{i}\right|$ denotes the measure of edges of the triangle $K$ and $\omega_{1}, \ldots, \omega_{k+1}$ denote the $k+1$ weights of the Gauss quadrature rule in the interval $\left[-\frac{1}{2}, \frac{1}{2}\right]$, which have the property $\omega_{1}+\cdots+\omega_{k+1}=1$.

As a first step, we intend to write the average $\bar{\phi}_{K}^{n+1}$ in (4.1) as

$$
\bar{\phi}_{K}^{n+1}=H\left(\phi_{1,1}^{\operatorname{int}(K)}, \phi_{1,2}^{\operatorname{int}(K)}, \ldots, \phi_{3, k+1}^{\operatorname{int}(K)}, \phi_{1,1}^{\operatorname{ext}(K)}, \phi_{1,2}^{\operatorname{ext}(K)}, \ldots, \phi_{3, k+1}^{\operatorname{ext}(K)}, \phi_{1}^{\operatorname{int}}, \ldots, \phi_{M}^{\text {int }}\right) .
$$

So we split $\bar{\phi}_{K}^{n}$ into point values of the DG polynomial $p_{K}(\boldsymbol{x})$ using a quadrature rule on the square $\left[-\frac{1}{2}, \frac{1}{2}\right]^{2}$ (the square is taken just for the purpose of showing the stability results). For simplicity in the expression we denote by $s_{1}, s_{2}$ and $s_{3}$ the three vertices of the triangle $K$ which is chosen in a counterclockwise order. We can represent the position vector $P$ of any point in the triangle $K$ in terms of barycentric coordinates as $P=\zeta_{1} s_{1}+\zeta_{2} s_{2}+\zeta_{3} s_{3}$. In the first step the square $\left[-\frac{1}{2}, \frac{1}{2}\right] \times\left[-\frac{1}{2}, \frac{1}{2}\right]$ taken in the $(u, v)$ plane is mapped on to the triangle by a set of functions, which are

$$
\begin{aligned}
& \boldsymbol{\varphi}_{1}(u, v)=\left(\frac{1}{2}+v\right) s_{1}+\left(\frac{1}{2}+u\right)\left(\frac{1}{2}-v\right) s_{2}+\left(\frac{1}{2}-u\right)\left(\frac{1}{2}-v\right) s_{3}, \\
& \boldsymbol{\varphi}_{2}(u, v)=\left(\frac{1}{2}-u\right)\left(\frac{1}{2}-v\right) s_{1}+\left(\frac{1}{2}+v\right) s_{2}+\left(\frac{1}{2}+u\right)\left(\frac{1}{2}-v\right) s_{3}, \\
& \boldsymbol{\varphi}_{3}(u, v)=\left(\frac{1}{2}+u\right)\left(\frac{1}{2}-v\right) s_{1}+\left(\frac{1}{2}-u\right)\left(\frac{1}{2}-v\right) s_{2}+\left(\frac{1}{2}+v\right) s_{3} .
\end{aligned}
$$


Each of the functions maps one of the sides of the square onto one vertex of $K$ and the other sides of the square to edges of the triangle $K$. Now we can write

$$
\bar{\phi}_{K}^{n}=\frac{1}{|K|} \int_{K} p_{K}(\boldsymbol{x}) \mathrm{d} \boldsymbol{x}=\frac{1}{|K|} \int_{-1 / 2}^{1 / 2} \int_{-1 / 2}^{1 / 2} p_{K}\left(\boldsymbol{\varphi}_{i}(u, v)\right)\left|\frac{\partial \boldsymbol{\varphi}_{i}(u, v)}{\partial(u, v)}\right| \mathrm{d} u \mathrm{~d} v \quad \text { for } i=1,2,3,
$$

where the determinant of the Jacobian $\partial \varphi_{i}(u, v) / \partial(u, v)$ can be easily computed as

$$
\left|\frac{\partial \varphi_{i}(u, v)}{\partial(u, v)}\right|=2|K|\left(\frac{1}{2}-v\right) .
$$

Let $\left\{v_{\beta}, \beta=1, \ldots, k+1\right\}$ denote $k+1$ Gauss quadrature points chosen on the interval $\left[-\frac{1}{2}, \frac{1}{2}\right]$ with the corresponding quadrature weights $\omega_{\beta}$. Also let $\left\{u_{\alpha}, \alpha=1, \ldots, N\right\}$ denote $N$ Gauss-Lobatto quadrature points chosen on the interval $\left[-\frac{1}{2}, \frac{1}{2}\right]$ with corresponding weight $\hat{\omega}_{\alpha}$, and where $N$ is the smallest integer such that $2 N-3 \geq k$. The tensor product of these two types of quadrature points are used to define the quadrature rule on the square $\left[-\frac{1}{2}, \frac{1}{2}\right] \times\left[-\frac{1}{2}, \frac{1}{2}\right]$. Therefore the quadrature points could be taken as $S_{K}=\left\{\left(u_{\alpha}, v_{\beta}\right), \alpha=1, \ldots, N, \beta=1, \ldots, k+1\right\}$, which yields exact quadrature for a polynomial $p(u, v)$ of degree less or equal $k$ in $u$, and of degree less or equal $2 k+1$ in $v$.

We now define the following polynomial of degree $k$ and $k+1$ in $u$ and $v$, respectively:

$$
\hat{p}_{K}^{i}(u, v)=p_{K}\left(\boldsymbol{\varphi}_{i}(u, v)\right)\left|\frac{\partial \boldsymbol{\varphi}_{i}(u, v)}{\partial(u, v)}\right|=2|K| p_{K}\left(\boldsymbol{\varphi}_{i}(u, v)\right)\left(\frac{1}{2}-v\right) .
$$

Then A.2 can be written as

$$
\bar{\phi}_{K}^{n}=\frac{1}{|K|} \sum_{\alpha=1}^{N} \sum_{\beta=1}^{k+1} \hat{p}_{K}^{i}\left(u_{\alpha}, v_{\beta}\right) \hat{\omega}_{\alpha} \omega_{\beta}=2 \sum_{\alpha=1}^{N} \sum_{\beta=1}^{k+1} p_{K}\left(\varphi_{i}\left(u_{\alpha}, v_{\beta}\right)\right)\left(\frac{1}{2}-v_{\beta}\right) \hat{\omega}_{\alpha} \omega_{\beta} .
$$

Now for each $i=1,2,3$ the set of points $\left\{\boldsymbol{\varphi}_{i}\left(S_{K}\right)\right\}$ acts as the set of quadrature points for the polynomial $p_{K}(\boldsymbol{x})$. Combination of these sets of quadrature points leads to a total of $3(N-1)(k+1)$ quadrature points, which we denote by $\hat{S}_{K}=\left\{\boldsymbol{\varphi}_{1}\left(S_{K}\right)\right\} \cup\left\{\boldsymbol{\varphi}_{2}\left(S_{K}\right)\right\} \cup\left\{\boldsymbol{\varphi}_{3}\left(S_{K}\right)\right\}$. In this way, the cell average in A.3 takes the form

$$
\begin{aligned}
\bar{\phi}_{K}^{n} & =\frac{1}{3} \sum_{i=1}^{3} \bar{\phi}_{K}^{n}=\frac{1}{3|K|} \sum_{i=1}^{3} \sum_{\alpha=1}^{N} \sum_{\beta=1}^{k+1} \hat{p}_{K}^{i}\left(u_{\alpha}, v_{\beta}\right) \hat{\omega}_{\alpha} \omega_{\beta} \\
& =\sum_{i=1}^{3} \sum_{\alpha=1}^{N} \sum_{\beta=1}^{k+1} p_{K}\left(\boldsymbol{\varphi}_{i}\left(u_{\alpha}, v_{\beta}\right)\right) \frac{2}{3}\left(\frac{1}{2}-v_{\beta}\right) \hat{\omega}_{\alpha} \omega_{\beta}=\sum_{\boldsymbol{x} \in \hat{S}_{K}} p_{K}(\boldsymbol{x}) \omega_{\boldsymbol{x}}
\end{aligned}
$$

where $\omega_{\boldsymbol{x}}$ is the quadrature weight corresponding to point $\boldsymbol{x} \in \hat{S_{K}}$. Consider now the quadrature points on the edges $\sigma_{i}$ of $K$. Note that $\boldsymbol{\varphi}_{1}(a), \boldsymbol{\varphi}_{2}(b)$ and $\boldsymbol{\varphi}_{3}(d)$ are mapped onto the edge $\sigma_{1}$ (see the sketch in Figure 10, but the side $a$ does not contain any quadrature points, and therefore the quadrature points on $\sigma_{1}$ are only $\varphi_{2}\left(\frac{1}{2}, v_{\beta}\right) \cup \varphi_{3}\left(-\frac{1}{2}, v_{\beta}\right)$. This implies that the quadrature points on $\sigma_{1}$ can be written in terms of barycentric coordinates as $\left(0, \frac{1}{2}+v_{\beta}, \frac{1}{2}-v_{\beta}\right)$ (which are the quadrature points $\left.\left\{\boldsymbol{\varphi}_{3}\left(-\frac{1}{2}, v_{\beta}\right)\right\}=\left\{\boldsymbol{\varphi}_{3}\left(-\frac{1}{2},-v_{\beta}\right)\right\}=\left\{\boldsymbol{\varphi}_{2}\left(\frac{1}{2}, v_{\beta}\right)\right\}\right)$. In a similar manner, the quadrature points associated to $\sigma_{2}$ are $\left(\frac{1}{2}-v_{\beta}, 0, \frac{1}{2}+v_{\beta}\right)$, and those on the edge $\sigma_{3}$ are $\left(\frac{1}{2}+v_{\beta}, \frac{1}{2}-v_{\beta}, 0\right)$.

The weight of the point $\left(0, \frac{1}{2}+v_{\beta}, \frac{1}{2}-v_{\beta}\right)$ is calculated as $\hat{\omega}_{1}=\hat{\omega}_{N}$, and thus

$$
\frac{1}{2}\left(\frac{1}{2}+v_{\beta}\right) \omega_{\beta} \hat{\omega}_{1}+\frac{2}{3}\left(\frac{1}{2}-v_{\beta}\right) \omega_{\beta} \hat{\omega}_{N}=\frac{2}{3} \omega_{\beta} \hat{\omega}_{1}
$$

Similarly, the weights on the edges $\sigma_{2}$ and $\sigma_{3}$ are computed as $\frac{2}{3} \omega_{\beta} \hat{\omega}_{1}$. 
Let us denote by $\phi_{i, \beta}^{\operatorname{int}(K)}$ and $\phi_{\nu}^{\text {int }}$ the values of the polynomial at the quadrature points on the edges and interior of the triangle $K$ respectively. Also the quadrature weights corresponds to the values $\phi_{\nu}^{\text {int }}$ is denoted by $\tilde{\omega}_{\nu}$. We have $M=3(N-2)(k+1)$ quadrature points in the interior of the triangle. Now the expression (A.4) becomes

$$
\bar{\phi}_{K}^{n}=\sum_{i=1}^{3} \sum_{\beta=1}^{k+1} \phi_{i, \beta}^{\operatorname{int}(K)} \frac{2}{3} \omega_{\beta} \hat{\omega}_{1}+\sum_{\nu=1}^{M} \phi_{\nu}^{\text {int }} \tilde{\omega}_{\nu},
$$

and we write the value of $\bar{\phi}_{K}^{n+1}$ as 4.2 . With these preliminaries we are now able to provide the missing proof of Lemma 4.1

Proof of Lemma 4.1. For ease of reference, we denote the right-hand side of 4.2 by $H(\ldots)$. We have

$$
H(\ldots)=\bar{\phi}_{K}^{n}-\frac{\Delta t}{|K|} \sum_{\beta=1}^{k+1} \omega_{\beta} \sum_{i=1}^{3} J\left(\phi_{i, \beta}^{\operatorname{int}(K)}, \phi_{i, \beta}^{\operatorname{ext}(K)}, \boldsymbol{u}_{\beta}^{i}, \boldsymbol{x}_{\beta}^{i}, \boldsymbol{n}_{K, \sigma_{i}}\right)\left|\sigma_{i}\right| .
$$

Using the conservativity of the numerical flux, we can assert that

$$
\begin{aligned}
& \sum_{i=1}^{3} J\left(\phi_{i, \beta}^{\operatorname{int}(K)}, \phi_{i, \beta}^{\operatorname{ext}(K)}, \boldsymbol{u}_{\beta}^{i}, \boldsymbol{x}_{\beta}^{i}, \boldsymbol{n}_{K, \sigma_{i}}\right)\left|\sigma_{i}\right| \\
& =J\left(\phi_{1, \beta}^{\operatorname{int}(K)}, \phi_{1, \beta}^{\operatorname{ext}(K)}, \boldsymbol{u}_{\beta}^{1}, \boldsymbol{x}_{\beta}^{1}, \boldsymbol{n}_{K, \sigma_{1}}\right)\left|\sigma_{1}\right|+J\left(\phi_{1, \beta}^{\operatorname{int}(K)}, \phi_{2, \beta}^{\operatorname{int}(K)}, \boldsymbol{u}_{\beta}^{1}, \boldsymbol{x}_{\beta}^{1},-\boldsymbol{n}_{K, \sigma_{3}}\right)\left|\sigma_{1}\right| \\
& \quad+J\left(\phi_{2, \beta}^{\operatorname{int}(K)}, \phi_{1, \beta}^{\operatorname{int}(K)}, \boldsymbol{u}_{\beta}^{1}, \boldsymbol{x}_{\beta}^{1}, \boldsymbol{n}_{K, \sigma_{1}}\right)\left|\sigma_{1}\right|+J\left(\phi_{2, \beta}^{\operatorname{int}(K)}, \phi_{2, \beta}^{\operatorname{ext}(K)}, \boldsymbol{u}_{\beta}^{2}, \boldsymbol{x}_{\beta}^{2}, \boldsymbol{n}_{K, \sigma_{2}}\right)\left|\sigma_{2}\right| \\
& \quad+J\left(\phi_{2, \beta}^{\operatorname{int}(K)}, \phi_{3, \beta}^{\operatorname{int}(K)}, \boldsymbol{u}_{\beta}^{3}, \boldsymbol{x}_{\beta}^{3}, \boldsymbol{n}_{K, \sigma_{3}}\right)\left|\sigma_{3}\right|+J\left(\phi_{3, \beta}^{\operatorname{int}(K)}, \phi_{2, \beta}^{\operatorname{int}(K)}, \boldsymbol{u}_{\beta}^{3}, \boldsymbol{x}_{\beta}^{3},-\boldsymbol{n}_{K, \sigma_{3}}\right)\left|\sigma_{3}\right| \\
& \quad+J\left(\phi_{3, \beta}^{\operatorname{int}(K)}, \phi_{3, \beta}^{\operatorname{ext}(K)}, \boldsymbol{u}_{\beta}^{3}, \boldsymbol{x}_{\beta}^{3}, \boldsymbol{n}_{K, \sigma_{3}}\right)\left|\sigma_{3}\right| .
\end{aligned}
$$

On the other hand, we have

$$
\begin{aligned}
H(\ldots)= & \sum_{i=1}^{3} \sum_{\beta=1}^{k+1} \phi_{i, \beta}^{\operatorname{int}(K)} \frac{2}{3} \omega_{\beta} \hat{\omega}_{1}+\sum_{\nu=1}^{M} \phi_{\nu}^{\operatorname{int}} \tilde{\omega}_{\nu}-\frac{\Delta t}{|K|} \sum_{\beta=1}^{k+1} \omega_{\beta} \sum_{i=1}^{3} J\left(\phi_{i, \beta}^{\operatorname{int}(K)}, \phi_{i, \beta}^{\operatorname{ext}(K)}, \boldsymbol{u}_{\beta}^{i}, \boldsymbol{x}_{\beta}, \boldsymbol{n}_{K, \sigma_{i}}\right)\left|\sigma_{i}\right| \\
= & \sum_{\nu=1}^{M} \phi_{\nu}^{\text {int }} \tilde{\omega}_{\nu}+\sum_{\beta=1}^{k+1} \frac{2}{3} \omega_{\beta} \hat{\omega}_{1} \sum_{i=1}^{3} \phi_{i, \beta}^{\operatorname{int}(K)} \\
& -\sum_{\beta=1}^{k+1} \frac{2}{3} \omega_{\beta} \hat{\omega}_{1}\left(\frac{3 \Delta t}{2|K| \hat{\omega}_{1}} \sum_{i=1}^{3} J\left(\phi_{i, \beta}^{\operatorname{int}(K)}, \phi_{i, \beta}^{\operatorname{ext}(K)}, \boldsymbol{u}_{\beta}^{i}, \boldsymbol{x}_{\beta}^{i}, \boldsymbol{n}_{K, \sigma_{i}}\right)\left|\sigma_{i}\right|\right) \\
= & \sum_{\nu=1}^{M} \phi_{\nu}^{\text {int }} \tilde{\omega}_{\nu}+\sum_{\beta=1}^{k+1} \frac{2}{3} \omega_{\beta} \hat{\omega}_{1}\left(H_{\beta}^{1}+H_{\beta}^{2}+H_{\beta}^{3}\right)
\end{aligned}
$$

where

$$
\begin{aligned}
H_{\beta}^{1}=\phi_{1, \beta}^{\operatorname{int}(K)}-\frac{3 \Delta t}{2 \hat{\omega}_{1}|K|} & {\left[J\left(\phi_{1, \beta}^{\operatorname{int}(K)}, \phi_{1, \beta}^{\operatorname{ext}(K)}, \boldsymbol{u}_{\beta}^{1}, \boldsymbol{x}_{\beta}^{1}, \boldsymbol{n}_{K, \sigma_{1}}\right)\left|\sigma_{i}\right|+J\left(\phi_{1, \beta}^{\operatorname{int}(K)}, \phi_{2, \beta}^{\operatorname{int}(K)}, \boldsymbol{u}_{\beta}^{1}, \boldsymbol{x}_{\beta}^{1},-\boldsymbol{n}_{K, \sigma_{1}}\right)\left|\sigma_{1}\right|\right], } \\
H_{\beta}^{2}=\phi_{2, \beta}^{\operatorname{int}(K)}-\frac{3 \Delta t}{2 \hat{\omega}_{1}|K|}\left[J\left(\phi_{2, \beta}^{\operatorname{int}(K)}, \phi_{1, \beta}^{\operatorname{int}(K)}, \boldsymbol{u}_{\beta}^{1}, \boldsymbol{x}_{\beta}^{1}, \boldsymbol{n}_{K, \sigma_{1}}\right)\left|\sigma_{1}\right|+J\left(\phi_{2, \beta}^{\operatorname{int}(K)}, \phi_{2, \beta}^{\operatorname{ext}(K)}, \boldsymbol{u}_{\beta}^{2}, \boldsymbol{x}_{\beta}^{2}, \boldsymbol{n}_{K, \sigma_{2}}\right)\left|\sigma_{2}\right|\right. & \\
& \left.\quad+J\left(\phi_{2, \beta}^{\operatorname{int}(K)}, \phi_{3, \beta}^{\operatorname{int}(K)}, \boldsymbol{u}_{\beta}^{3}, \boldsymbol{x}_{\beta}^{3}, \boldsymbol{n}_{K, \sigma_{3}}\right)\left|\sigma_{3}\right|\right] \\
H_{\beta}^{3}=\phi_{3, \beta}^{\operatorname{int}(K)}-\frac{3 \Delta t}{2 \hat{\omega}_{1}|K|} & {\left[J\left(\phi_{3, \beta}^{\operatorname{int}(K)}, \phi_{2, \beta}^{\operatorname{int}(K)}, \boldsymbol{u}_{\beta}^{3}, \boldsymbol{x}_{\beta}^{3},-\boldsymbol{n}_{K, \sigma_{3}}\right)\left|\sigma_{3}\right|+J\left(\phi_{3, \beta}^{\operatorname{int}(K)}, \phi_{3, \beta}^{\operatorname{ext}(K)}, \boldsymbol{u}_{\beta}^{3}, \boldsymbol{x}_{\beta}^{3}, \boldsymbol{n}_{K, \sigma_{3}}\right)\left|\sigma_{3}\right|\right] . }
\end{aligned}
$$

The term $H_{\beta}^{1}$ can be viewed as a function of $\phi_{1, \beta}^{\operatorname{int}(K)}, \phi_{1, \beta}^{\operatorname{ext}(K)}$ and $\phi_{2, \beta}^{\operatorname{int}(K)}$. Therefore, differentiating $H_{\beta}^{1}$ with respect to these variables and applying the CFL condition 4.3 , we verify that $H_{\beta}^{1}$ is increasing 
in each of the arguments $\phi_{1, \beta}^{\operatorname{int}(K)}, \phi_{1, \beta}^{\operatorname{ext}(K)}$ and $\phi_{2, \beta}^{\operatorname{int}(K)}$. Similar arguments can be applied to $H_{\beta}^{2}$ and $H_{\beta}^{3}$. In view of equation (A.6) it follows that $H$ is an increasing function in each of its arguments appearing in 4.2 .

\section{References}

[1] Adimurthi, J. Jaffré, G.D.V. Gowda, Godunov-type methods for conservation laws with a flux function discontinuous in space, SIAM J. Numer. Anal. 42 (2004) 179-208.

[2] Adimurthi, J. Jaffré, G.D.V. Gowda, The DFLU flux for systems of conservation laws, J. Comput. Appl. Math. 247 (2013) 102-123.

[3] V. Anaya, G.N. Gatica, D. Mora, R. Ruiz-Baier, An augmented velocity-vorticity-pressure formulation for the Brinkman problem, Int. J. Numer. Meth. Fluids 79 (2015) 109-137.

[4] V. Anaya, D. Mora, R. Oyarzúa, R. Ruiz-Baier, A priori and a posteriori error analysis of a fullymixed scheme for the Brinkman problem, Numer. Math., (2015) in press.

[5] V. Anaya, D. Mora, C. Reales, R. Ruiz-Baier, Stabilized mixed approximation of axisymmetric Brinkman flows, ESAIM: Math. Model. Numer. Anal. 49 (2015) 855-874.

[6] G.E. Barter, D.L. Darmofal, Shock capturing with PDE-based artificial viscosity for DGFEM. I: formulation, J. Comput. Phys. 229 (2010) 1810-1827.

[7] P. Bastian, A fully-coupled discontinuous Galerkin method for two-phase flow in porous media with discontinuous capillary pressure, Comput. Geosci. 18 (2014) 779-796.

[8] C. Bi, J. Geng, Discontinuous finite volume element methods for parabolic problems, Numer. Meth. Partial Diff. Eqns. 26 (2010) 367-383.

[9] C. Bi, M. Liu, A discontinuous finite volume element method for second-order elliptic problems, Numer. Meth. Part. Diff. Eqns. 28 (2012) 425-440.

[10] R. Biswas, K.D. Devine, J. Flaherty, Parallel, adaptive finite element methods for conservation laws, Appl. Numer. Math. 14 (1994) 255-283.

[11] R. Bürger, K.H. Karlsen, H. Torres, J.D. Towers, Second-order schemes for conservation laws with discontinuous flux modelling clarifier-thickener units, Numer. Math. 116 (2010) 579-617.

[12] R. Bürger, S. Kumar, R. Ruiz-Baier, Discontinuous finite volume element discretization for coupled flow-transport problems arising in models of sedimentation, J. Comput. Phys. 299 (2015) 446-471.

[13] R. Bürger, R. Ruiz-Baier, K. Schneider, M. Sepúlveda, Fully adaptive multiresolution schemes for strongly degenerate parabolic equations with discontinuous flux, J. Engrg. Math. 60 (2008) 365-385.

[14] R. Bürger, R. Ruiz-Baier, H. Torres, A stabilized finite volume element formulation for sedimentation-consolidation processes, SIAM J. Sci. Comput. 34 (2012) B265-B289.

[15] A. Burbeau, P. Sagaut, C.H. Bruneau, A problem-independent limiter for high-order Runge-Kutta discontinuous Galerkin methods, J. Comput. Phys. 169 (2001) 111-150.

[16] J. Camaño, R. Oyarzúa, G.N. Gatica, R. Ruiz-Baier, P. Venegas, New fully-mixed finite element methods for the Stokes-Darcy coupling, Comput. Meth. Appl. Mech. Engrg. 295 (2015) 362-395.

[17] H. Class, R. Helmig, J. Niessner, U. Ölmann, Multiphase Processes in Porous Media, In: Multifield Problems in Solid and Fluid Mechanics Lecture Notes in Applied and Computational Mechanics Volume 28, 2006, pp 45-82. 
[18] B. Cockburn, S. Hou, C.-W. Shu, The Runge-Kutta local projection discontinuous Galerkin finite element method for conservation laws. IV. The multidimensional case, Math. Comp. 54 (1990) 545581.

[19] B. Cockburn, C.-W. Shu, The Runge-Kutta discontinuous Galerkin method for conservation laws V: Multidimensional systems, J. Comput. Phys. 141 (1998) 199-224.

[20] B. Cockburn, C.-W. Shu, TVB Runge-Kutta local projection discontinuous Galerkin finite element method for conservation laws II: General framework, Math. Comp. 52 (1989) 411-435.

[21] G.M. Coclite, S. Mishra, N.H. Risebro, F. Weber, Analysis and numerical approximation of Brinkman regularization of two-phase flows in porous media, Comput. Geosci. 18 (2014) 637-659.

[22] L.J. Durlofsky, Y. Efendiev, V. Ginting, An adaptive local-global multiscale finite volume element method for two-phase flow simulations, Adv. Water Res. 30 (2007) 576-588.

[23] P. Frolkovič, H. De Schepper, Numerical modelling of convection dominated transport coupled with density driven flow in porous media, Adv. Water Res. 24 (2001) 63-72.

[24] A. Fumagalli, A. Scotti, A numerical method for two-phase flow in fractured porous media with non-matching grids. Adv. Water Res. 62(C) (2013) 454-464.

[25] F. Furtado, V. Ginting, F. Pereira, M. Presho, Operator splitting multiscale finite volume element method for two-phase flow with capillary pressure, Transp. Porous Media 90 (2011) 927-947.

[26] S. Gottlieb, On high order strong stability preserving Runge-Kutta and multi step time discretizations, J. Sci. Comput. 25 (2005) 105-128.

[27] S. Gottlieb, C.-W. Shu, Total variation diminishing Runge-Kutta schemes, Math. Comp. 67 (1998) $73-85$.

[28] W. Jäger, A. Mikelic, On the interface boundary condition of Beavers, Joseph and Saffmann, SIAM J. Appl. Math. 60 (2000) 1111-1127.

[29] K. Konstantinos, P. Konstantinos, E. John, A limiting approach for DG discretizations on mixed type meshes, Comput. Methods Appl. Mech. Engrg. 285 (2015), 587-620.

[30] S. Kumar, A mixed and discontinuous Galerkin finite volume element method for incompressible miscible displacement problems in porous media, Numer. Meth. Partial Diff. Eqns. 28 (2012) 13541381.

[31] S. Kumar, On the approximation of incompressible miscible displacement problems in porous media by mixed and standard finite volume element methods, Int. J. Model. Simul. Sci. Comput. (2013) 4:paper 1350013 (30pp).

[32] S. Kumar, N. Nataraj, A.K. Pani, Discontinuous Galerkin finite volume element methods for second order linear elliptic problems, Numer. Meth. Part. Diff. Eqns. 25 (2009) 1402-1424.

[33] S. Kumar, R. Ruiz-Baier, Equal order discontinuous finite volume elements for the Stokes problem, J. Sci. Comput. 65 (2015) 956-978.

[34] D. Kuzmin, Slope limiting for discontinuous Galerkin approximations with a possibly non-orthogonal Taylor basis, Internat. J. Numer. Methods Fluids 71 (2013), 1178-1190.

[35] K. Sudarshan Kumar, C. Praveen, G.D.V. Gowda, A finite volume method for a two-phase multicomponent polymer flooding, J. Comput. Phys. 275 (2014) 667-695. 
[36] R.D. Lazarov, S.Z. Tomov, Adaptive finite volume element method for convection-diffusion-reaction problems in 3-D. In: P. Minev, Y. Lin (Eds.), Scientific Computing and Applications, Nova Sci. Publish. Inc., NY, USA (2001), pp. 91-106.

[37] Z. Luo, H. Li, P. Sun, A fully discrete stabilized mixed finite volume element formulation for the non-stationary conduction-convection problem, J. Math. Anal. Appl. 404 (2013) 71-85.

[38] S. Osher, C.-W. Shu, Efficient implementation of essentially non-oscillatory shock-capturing schemes, J. Comput. Phys. 77 (1988) 439-471.

[39] R. Rannacher, S. Turek, Simple nonconforming quadrilateral Stokes element, Numer. Meth. Partial Diff. Eqns. 8 (1992) 97-111.

[40] R. Ruiz-Baier, I. Lunati, Mixed finite element - primal finite volume element discretization of multicontinuum models, (2015) submitted.

[41] R. Ruiz-Baier, H. Torres, Numerical solution of a multidimensional sedimentation problem using finite volume-elements, Appl. Numer. Math. 95 (2015) 280-291.

[42] C.-W. Shu, Discontinuous Galerkin method for time-dependent problems: survey and recent developments. In X. Feng, O. Karakashian and Y. Xing (Eds.), Recent Developments in Discontinuous Galerkin Finite Element Methods for Partial Differential Equations, Springer International Publishing, Cham, Switzerland (2014), 25-62.

[43] A. Suresh, H.T. Huynh, Accurate monotonicity-preserving schemes with Runge-Kutta time stepping, J. Comput. Phys. 136 (1997) 83-99.

[44] M.F. Wheeler, T. Wick, W. Wollner, An augmented-Lagrangian method for the phase-field approach for pressurized fractures, Comput. Meth. Appl. Mech. Engrg. 271 (2014) 69-85.

[45] Z.L. Xu, X.-Y. Chen, Y.J. Liu, A New Runge-Kutta discontinuous Galerkin method with conservation constraint to improve CFL condition for solving conservation laws, J. Comput. Phys. 278 (2014) $348-377$.

[46] X. Ye, A discontinuous finite volume method for the Stokes problem, SIAM J. Numer. Anal. 44 (2006) 183-198.

[47] X. Zhang, Y. Xia, C.-W. Shu, Maximum-principle-satisfying and positivity-preserving high order discontinuous Galerkin schemes for conservation laws on triangular meshes, J. Sci. Comput. 50 (2012) 29-62.

[48] X. Zhang, C.-W. Shu, On maximum principle satisfying high order schemes for scalar conservation laws, J. Comput. Phys. 229 (2010) 3091-3120. 